Hohlfeld, B. F.; Gitter, B.; Flanagan, K. J.; Kingsbury, C. J.; Kulak, N.; Senge, M. O.; Wiehe A. (2020):

Exploring the relationship between structure and activity in BODIPYs designed for antimicrobial phototherapy.

Organic \& Biomolecular Chemistry, 18, 2416-2431. doi: 10.1039/D0OB00188K

ARTICLE

\title{
Exploring the Relationship Between Structure and Activity in BODIPYs Designed for Antimicrobial Phototherapy
}

Received 00th January 20xx, Accepted 00th January 20xx

DOI: $10.1039 / x 0 x \times 00000 x$

\author{
Benjamin F. Hohlfeld ${ }^{a, b, d}$, Burkhard Gitter ${ }^{d}$, Keith J. Flanagan ${ }^{c}$, Christopher J. Kingsbury $^{c}$, Nora Kulak \\ b, Mathias O. Senge ${ }^{c}$ and Arno Wiehe ${ }^{* a, d}$
}

\begin{abstract}
A synthetic strategy to BODIPY dyes is presented giving access to a range of new compounds relevant in the context of antimicrobial photodynamic therapy (aPDT). BODIPYs with the 8-(4-fluoro-3-nitrophenyl) and the 8-pentafluorophenyl substituent were used for the synthesis of new mono- and dibrominated BODIPYs. The para-fluorine atoms in these electronwithdrawing groups facilitate functional modification via nucleophilic aromatic substitution $\left(\mathrm{S}_{N} A r\right)$ with a number of amines and thio-carbohydrates. Subsequently, the antibacterial phototoxic activity of these BODIPYs has been assessed in bacterial assays against the Gram-positive germ S. aureus and also against the Gram negative germ $P$. aeruginosa. The bacterial assays allowed to identify substitution patterns which ensured antibacterial activity not only in phosphate-buffered saline (PBS) but also in the presence of serum, hereby more realistically modelling the complex biological environment that is present in clinical applications.
\end{abstract}

\section{Introduction}

Rising drug and multi-drug resistance of bacteria and microorganisms against common antibiotics is a serious problem for public health systems around the world. In 2014 the World Health Organization (WHO) published a report on the surveillance of antimicrobial resistance in bacterial and viral cultures, indicating that in the coming decades the effectiveness of common antimicrobial drugs will be severely affected. Therefore, new strategies against microbial pathogens and antimicrobial resistance need to be developed. ${ }^{1}$ Antimicrobial photodynamic therapy (aPDT) represents such a new strategy and shows large potential as an alternative to common antibiotic treatments. ${ }^{2}$ aPDT is a light-based treatment for the inactivation of bacteria. The antimicrobial effect of aPDT is based on the photoactivation of a dye (photosensitizer) by light of an appropriate wavelength. In the presence of oxygen, cytotoxic reactive oxygen species (ROS) are generated by the excited photosensitizer, which finally results in the oxidative cellular destruction of microorganisms. ${ }^{2 a, 3}$ aPDT is not limited to an application against bacteria, but can as well be used for the inactivation of viruses ${ }^{4}$, fungi ${ }^{5}$, and protozoae. ${ }^{6}$

\footnotetext{
a. Institut für Chemie u. Biochemie, Freie Universität Berlin Takustr. 3, 14195 Berlin, Germany.

b. Institut für Chemie und Biochemie, Freie Universität Berlin, Fabeckstr. 34/36, 14195 Berlin, Germany.

Medicinal Chemistry, Trinity Translational Medicine Institute, Trinity Centre for Health Sciences, Trinity College Dublin, The University of Dublin, St James's Hospital, Dublin 8, Ireland.

d. biolitec research $\mathrm{GmbH}$, Otto-Schott-Str. 15, 07745 Jena, Germany.

Electronic Supplementary Information (ESI) available: See DOI: 10.1039/x0xx00000x
}

Also, antimicrobial coatings with photoactive substances have found interest in the protection of surfaces against bacterial colonization and biofilms. ${ }^{7}$ These coatings are not limited to medical applications, like the disinfection of surfaces in hospitals ${ }^{8}$ or antimicrobial coatings for implants, ${ }^{9}$ but as well antimicrobial surfaces are relevant for protecting water pipelines and surfaces in the food industry against colonization with microorganisms. ${ }^{7,10}$ In this context, borondipyrromethenes (BODIPYs) show potential for antimicrobial inactivation ${ }^{11}$ and have been used as components of antimicrobial coatings. ${ }^{12}$ So far, BODIPYs are well established as fluorescence imaging dyes in diagnostics ${ }^{13}$ and share many characteristics with well-known photosensitizers, e.g., porphyrins and chlorins, such as their intense color and fluorescence, high extinction coefficients, and resistance against photobleaching. ${ }^{14}$ However, the basic BODIPY structure itself only shows a low ability for generating ROS and a low phototoxicity, making such structures ideal for fluorescence and imaging applications. Therefore, in order to be suitable for aPDT the BODIPY structure needs to be modified, e.g., to increase excited triplet state and ROS formation, ${ }^{15}$ to adjust solubility to the biological environment, ${ }^{16}$ and to increase interaction with bacterial target structures. ${ }^{17}$ Especially, the addition of heavy atoms (e.g., bromine or iodine) into the 2- and 6-positions of the BODIPY core structure has been shown to change the BODIPYs photochemical properties in a favorable way and leads to BODIPY derivates in principle suitable for an application in aPDT. ${ }^{18}$ This 'heavy atom effect' dramatically impacts the BODIPY's photochemical properties, shifting the absorption maximum towards higher wavelengths (500 to $600 \mathrm{~nm}$ ) and leading to an increased population of the excited triplet state. The latter is caused by enhancement of the spin-orbital coupling and improvement of the intersystem crossing rate. ${ }^{15 \mathrm{~g}, 18 \mathrm{c}, 19}$ Of course, also the other positions of the BODIPY can be used to modify its 
photochemical and biological properties. ${ }^{15 f, 20}$ Specifically, the substitution in the 8- or meso-position can be used to introduce a multitude of functionalities but also to change the photochemical properties. For example, heavy atom free 8-anthra, -phenanthreneand -pyrene-BODIPYs efficiently generate triplet excited states via photoinduced electron transfer ${ }^{21 a, 21 b}$ and increased singlet oxygen quantum yields have been reported for meso- $p$-aminophenyl substituted BODIPYs ${ }^{21 c}$

In this work the stepwise optimization of the 8-pentafluorophenyl BODIPY and the 8-(4-fluoro-3-nitrophenyl) BODIPY for an application in antimicrobial phototherapy is presented. The BODIPY core structure or its methylated congeners allows for targeted halogenation, while the pentafluorophenyl moiety and the 4-fluoro3-nitrophenyl moiety on the other hand enable a variety of subsequent nucleophilic substitutions. Thus, targeted bromination of the 2-position (monobromination) and the 2,6-positions (dibromination) of the BODIPYs and as well the $p$-fluorine exchange with numerous nucleophiles, amines and thio-carbohydrates, were applied to synthesize specifically functionalized BODIPYs. With these methods four series of BODIPYs were synthesized: BODIPYs with no core substitution, core-tetramethyl-substituted BODIPYs, tetramethyl-dibromo-substituted BODIPYs, and monobrominated BODIPYs. These groups of BODIPYs were evaluated for their antibacterial effect with and without light in assays against $S$. aureus. Moreover, instead of testing the substances in phosphate-buffered saline (PBS) only, all tests were additionally carried out with the addition of horse serum, to challenge their activity under more realistic conditions, modelling the complex mixtures that are present in clinical applications like chronic wounds. ${ }^{22}$ In these assays, structures with a high bactericidal and phototoxic potential against S. aureus were identified, pointing at structural elements that favor antibacterial activity.

\section{Results and discussion}

\section{Synthesis of target compounds}

In the literature the stepwise halogenation of the BODIPY core structure with different halogens has been described. Specifically, brominated and iodinated BODIPYs are attractive in eliciting the desired heavy atom effect. ${ }^{19}$ For the synthesis of such brominated BODIPYs, different synthetic pathways have been reported, e.g. using molecular bromine, ${ }^{23}$ copper(II) bromide, ${ }^{24}$ or $\mathrm{N}$ bromosuccinimide (NBS). ${ }^{25}$ As target structures for the selective bromination BODIPYs with an 8-pentafluorophenyl or an 8-(4-fluoro3-nitrophenyl) moiety were chosen. The pentafluorophenyl group, ${ }^{26}$ as well as the 4-fluoro-3-nitrophenyl group, ${ }^{27}$ have shown great potential for nucleophilic aromatic substitution reactions $\left(\mathrm{S}_{N} \mathrm{Ar}\right)$, e.g., introducing sugar moieties ${ }^{28}$ or alkynyl groups giving access to subsequent 1,3-dipolar cycloaddition reactions for connection with biomolecules ("click" chemistry). ${ }^{29}$

In preparation for the following investigations, BODIPYs 1a, 3a, 4a, and $6 a-12 a$ were synthesized according to the literature (Scheme 1) from their corresponding pre-functionalized dipyrromethanes $\mathbf{1}$, 3, 4, and 6 - 12. ${ }^{26 a, 26 b, 27 a}$ The new BODIPYs 2a and 5a were synthesized analogously from dipyrromethanes $\mathbf{2}$ and $\mathbf{5}$ and were obtained in $16 \%$ and $11 \%$ yield, respectively (for details see Supporting Information).

First, the targeted halogenation of the 2- and 6- position was investigated with BODIPYs 1a, 7a, 8a, and 9a. The reactions were performed according to the description in the literature, using 1,1,1,3,3,3-hexafluoro-2-propanol (HFIP) as solvent and NBS as halogen source. ${ }^{25}$ The BODIPYs were dissolved in HFIP and 2.5 equiv. of NBS were added. Within a short reaction time, the starting material was no longer detectable by TLC. However, instead of the favored 2,6-dibromo-substituted product a complex mixture of multiple BODIPYs was observed (Scheme 2, variant A). Next to the mono- and dihalogenated BODIPYs, other BODIPYs were detected.

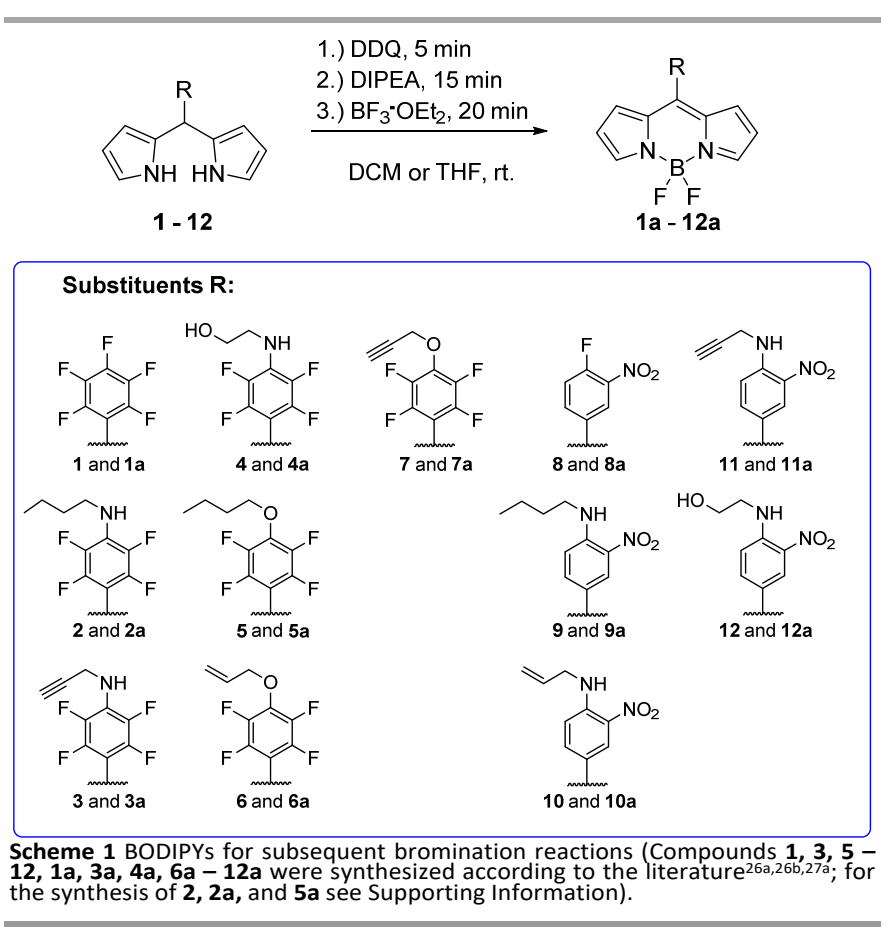

Variant A:

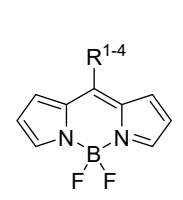

1a, $7 a-9 a$

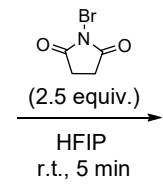

(n)

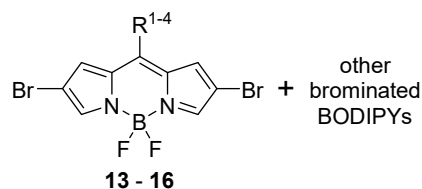

$13-16$ 15: $R^{3} \quad 16: R^{4}$

Variant B:

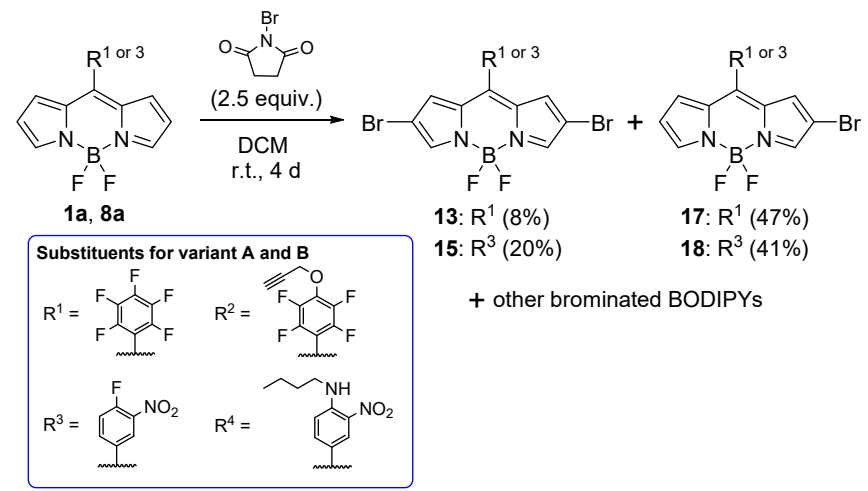

Scheme 2 Bromination of BODIPYs with NBS. 
In order to obtain the desired 2,6-dibrominated BODIPYs, the synthesis was modified as follows. Prasannan et al. showed that in dichloromethane (DCM) much longer reaction times $(15-17 \mathrm{~h})$ were required for a targeted 2,6-bromination, in comparison to HFIP as solvent, with the 2,6-dibromated BODIPY being the favorite product. ${ }^{30}$ Based on this, BODIPY $1 \mathrm{a}$ and the 8 a were reacted under these modified conditions (DCM as solvent and 2.5 equiv. of NBS, Scheme 2, variant B). Interestingly, after $24 \mathrm{~h}$ larger amounts of the starting material could still be detected via TLC. Finally, after $4 \mathrm{~d}$ the starting material was no longer detectable and the expected 2,6dibromo products $\mathbf{1 3}$ and $\mathbf{1 5}$ could be isolated. However, the desired BODIPYs $\mathbf{1 3}$ and $\mathbf{1 5}$ were only obtained as the minor product. Next to the desired dibrominated BODIPYs, other BODIPYs were as well detected via TLC. The major products were identified as the corresponding monobrominated BODIPYs $\mathbf{1 7}$ and $\mathbf{1 8}$ (for details see Supporting Information). Thus, a targeted monobromination of the 2-position of the BODIPYS seemed possible with NBS in DCM and adequate reaction times. Therefore, pre-functionalized BODIPYs were dissolved in DCM and treated with 2.5 equiv. of NBS (Table 1 ) to obtain the monobrominated BODIPYs $\mathbf{1 7} \mathbf{- 2 4}$. The reaction times for monobromination were similar in all cases (1-3 $h$, Table 1 ), with a tendency of the tetrafluorophenyl-substituted compounds requiring shorter reaction times (Table 1, entry 1-5, respectively). BODIPYs 17 and 18 were obtained in high yields (Table 1 ). A number of BODIPYs carrying side chains $(\mathbf{1 9}, \mathbf{2 0}$, and $\mathbf{2 3})$ still provide good to high yields (Table 1). In the case of BODIPY 21, 22, and $\mathbf{2 4}$ - carrying the prop-2enyl and the prop-2-ynyloxy moieties - only moderate yields were observed (Table 1). Despite this synthesis of the monobrominated compounds, in some cases the reaction mixtures required tedious column chromatography, as the mono substituted BODIPY and the starting material showed nearly the same elution behavior. In addition, there were hints that next to the mono- and dihalogenation of the BODIPY core structure again, like for HFIP as solvent, other positions of the BODIPY were substituted as well with bromine, leading to a mixture of BODIPYs and resulting in complicated purifications. Such multiple brominations have also been reported for other BODIPYs. ${ }^{19 a, 23}$

To exclude the substitution with bromine in other than the desired 2- and 6-positions, the synthetic concept was modified and the investigations were continued with 1,3,5,7-tetramethyl substituted BODIPYs. By 'blocking' the 1,3,5, and 7-positions of the BODIPY with methyl groups, the halogenation should be restricted to the 2- and 6-position. The synthesis of such 1,3,5,7-tetramethyl substituted BODIPYs is known in the literature. ${ }^{31}$ Hence, BODIPY 25, carrying the pentafluorophenyl substituent was prepared according to the literature. ${ }^{32}$ This synthetic procedure was also used to prepare the analogous BODIPY $\mathbf{2 6}$ with the 4-fluoro-3-nitrophenyl moiety (Scheme 3). 4-Fluoro-3-nitrobenzaldehyde and 2,4-dimethylpyrrole were dissolved in DCM and were reacted under TFA catalysis, followed by oxidation with $\mathrm{DDQ}$ and $\mathrm{BF}_{3}$ complexation, to obtain BODIPY 26 in $47 \%$ yield (Scheme 2). In the next step, BODIPYs 25 and $\mathbf{2 6}$ were modified in the para-position of their phenyl moieties via $\mathrm{S}_{N} \mathrm{Ar}$ with several different amines (Tables 2 and 3 ). The functionalization of BODIPY $\mathbf{2 5}$ with a limited number of amines and thiols has already been described in the literature. ${ }^{32}$
Table 1 Targeted monobromination of BODIPYs with NBS.

\begin{tabular}{|c|c|c|c|c|c|}
\hline $1 \mathrm{a}, 3 \mathrm{a}^{\prime}$ & $\begin{array}{l}N=1 \\
=10 a\end{array}$ & 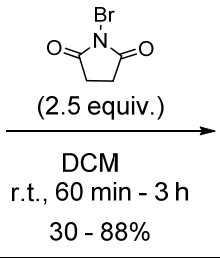 & - & & \\
\hline Entry & $\begin{array}{l}\text { Starting } \\
\text { material }\end{array}$ & Substituent (R) & Product & $\begin{array}{l}\text { Time } \\
{[\mathrm{h}]}\end{array}$ & $\begin{array}{l}\text { Yield } \\
{[\%]}\end{array}$ \\
\hline 1 & $1 a$ & & 17 & 2.5 & 84 \\
\hline 2 & $3 a$ & & 19 & 1.5 & 63 \\
\hline 3 & $5 a$ & & 20 & 1 & 53 \\
\hline 4 & $6 a$ & & 21 & 1 & 31 \\
\hline 5 & $7 a$ & & 22 & 1 & 34 \\
\hline 6 & $8 a$ & & 18 & 3 & 64 \\
\hline 7 & $9 a$ & & 23 & 3 & 48 \\
\hline 8 & 10a & & 24 & 3 & 30 \\
\hline
\end{tabular}

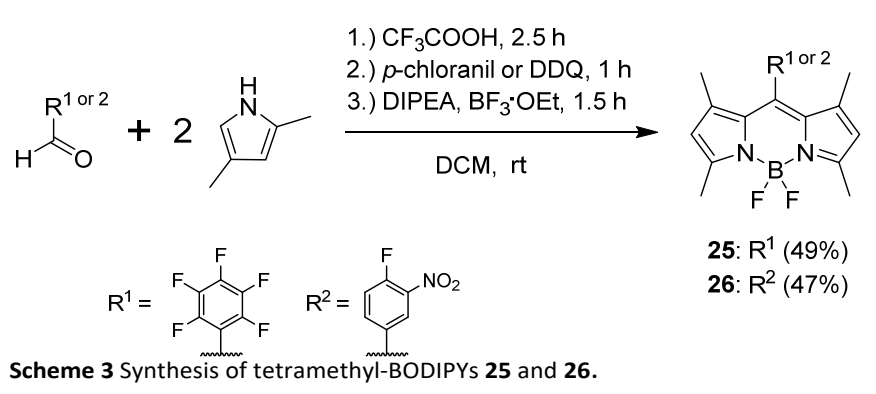

Based on the $p$-fluorine exchange reaction with $n$-butylamine described by Vives and co-workers ${ }^{32}$, the $S_{N} A r$ with other amines was performed (Table 2). BODIPY 25 was dissolved in DMF and treated with an excess of the corresponding primary amine. The desired amino-substituted BODIPYs $\mathbf{2 7}$ - $\mathbf{2 9}$ were obtained in good to moderate yields, without need for heating or catalytic support. The substitutions were complete within a time period of $4 \mathrm{~h}$ (Table 2 , 
entry 1-3). In the case of the nucleophilic substitution with serinol; however, the desired product could not be obtained (Table 2, entry 4). Based on previous results carried from our group on $S_{N} A r$ with 4fluoro-3-nitrophenyldipyrromethanes and 4-fluoro-3-nitrophenylporphyrins, ${ }^{27 a}$ the nucleophilic substitutions were now extended to BODIPY 26. For this, BODIPY 26 was dissolved in DCM or DMSO and reacted with an excess of the corresponding amine at room temperature (Table 3). All desired BODIPYs $(\mathbf{3 0} \mathbf{- 3 3})$ were formed as expected in moderate to high yields, again without need for heating or catalytic support. In the nucleophilic substitutions of $\mathbf{2 6}$, yields over $80 \%$ were achieved with $n$-butylamine and ethanolamine as nucleophiles (Table 3, entry 1 and 3 ). The substitution with propargylamine led to the desired product in a moderate yield (Table 3 , entry 2). The reaction was also successful with a solid amine as exemplified by the reaction of $\mathbf{2 6}$ with serinol (Table 3, entry 4).

Table 2 Nucleophilic aromatic substitutions of BODIPY 25.

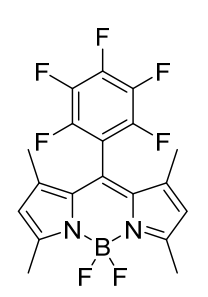

25

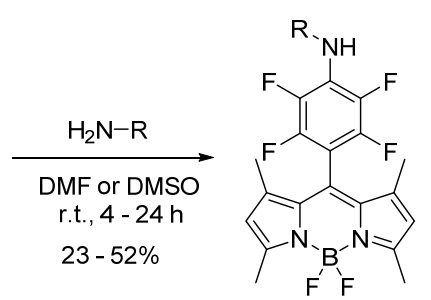

$27-29$

\begin{tabular}{llllll}
\hline Entry & $\begin{array}{l}\text { Substituent } \\
(\mathrm{R})\end{array}$ & Product & $\begin{array}{l}\text { Time } \\
{[\mathrm{h}]}\end{array}$ & Solvent & $\begin{array}{l}\text { Yield } \\
{[\%]}\end{array}$ \\
\hline $1^{\mathrm{a}}$ & & $\mathbf{2 7}$ & 4 & DMF & 52 \\
2 & $\mathbf{2 8}$ & 4 & DMF & 23 \\
3 & $\mathbf{2 9}$ & 4 & DMF & 41 \\
$4^{\mathrm{b}}$ & & & 24 & DMSO & -
\end{tabular}

a BODIPY was prepared according to the literature. ${ }^{32}$

b No Product was obtained (see Supporting Information).

Table 3 Nucleophilic aromatic substitutions of BODIPY 26.

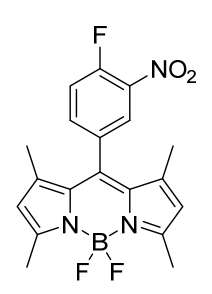

26

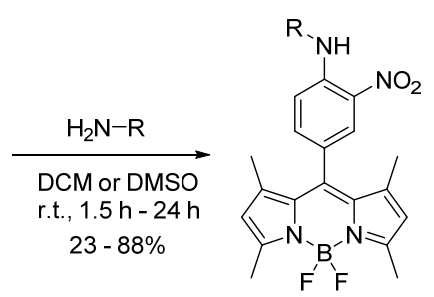

$30-33$

\begin{tabular}{llllll}
\hline Entry & $\begin{array}{l}\text { Substituent } \\
(\mathrm{R})\end{array}$ & Product & $\begin{array}{l}\text { Time } \\
{[\mathrm{h}]}\end{array}$ & Solvent & $\begin{array}{l}\text { Yield } \\
{[\%]}\end{array}$ \\
\hline 1 & & 30 & 1.5 & DCM & 88 \\
2 & 31 & 24 & DCM & 44 \\
3 & 32 & 24 & DCM & 87 \\
4 & 33 & 24 & DMSO & 23
\end{tabular}

For BODIPYs 11a and $\mathbf{2 9}$ crystals suitable for X-ray single crystal structure determination were obtained. Figure 1 shows the structure of 11a which was solved in a triclinic space group with one molecule present in the asymmetric unit (see also Supporting Information; Figure S1). One important feature is the tilt angle, which describes the orientation of the phenyl ring relative to the BODIPY framework. For 11a the tilt angle for these two structural elements is $39.2(2)^{\circ}$ similar to our previously reported structure. ${ }^{27 a}$ The second noteworthy feature in the structure of $11 \mathbf{a}$ is the presence of an intramolecular hydrogen bond between $\mathrm{N}(2)-\mathrm{H}(2) \cdots \mathrm{O}(2)[2.6256(11)$ $\left.\AA \mathrm{N} \cdots \mathrm{O}, 129.9(12)^{\circ}\right]$ (Figure 1). This bond holds the nitro group in a co-planar conformation with regards to the benzene ring. Unlike our previously reported structure, there is little indication of any additional interactions involving the nitro group. Instead, there are several interactions seen between the fluorine atoms with the propargyl and benzene moieties. The first of these interactions are shown in Figure 2, where the two interactions centered on the propargyl group are the head-to-tail linear network (F13) $\{C(21)-$ $\left.\mathrm{H}(21 \mathrm{~A}) \cdots \mathrm{F}(13)\left[2.9835(11) \AA \mathrm{C} \cdots \mathrm{F}, 126.2(7)^{\circ}\right]\right\}$ and the head-to-tail stacked (F14) dimers $\{\mathrm{C}(23)-\mathrm{H}(23) \cdots \mathrm{F}(14)[3.2348(12) \AA \mathrm{C} \cdots \mathrm{F}$, $\left.\left.156.9(2)^{\circ}\right]\right\}$. The final intermolecular interaction in this structure is a head-to-head contact seen between the fluorine atom F14 and the benzene hydrogen atom $\mathrm{H} 20\{\mathrm{C}(20)-\mathrm{H}(2) \cdots \mathrm{F}(14)[3.3097(11) \AA \AA \mathrm{C} \cdots \mathrm{F}$, $\left.\left.161.3(6)^{\circ}\right]\right\}$ which holds two BODIPY cores in a stacked dimer formation with a centroid-to-centroid distance of 4.332(7) $\AA$, and plane-to-plane shift of 2.514(9) ^ (See Supporting Information; Figure S2). These motifs result in a complex packing pattern shown in Figure S3 (see Supporting Information). Figure 3 shows the structure of 29, which was solved in the orthorhombic space group $P c a 2_{1}$. The asymmetric unit of this crystal structure contains one molecule of 29. Bond distances were indicative of the assigned structure with delocalized $\pi$-bonding, with a large difference in the two B-F distances of the BODIPY unit, at B(4)-F(18) 1.381(3) $\AA, B(4)-$ $F(17) 1.414(3) \AA$. In contrast to $11 a$, the tetrafluorophenyl rings at the 8-position of $\mathbf{2 9}$ are oriented approximately perpendicular to the BODIPY plane, at an angle of $89.68(6)^{\circ}$, due to additional steric bulk from methyl groups present at the 1 and 7 positions of the BODIPY.

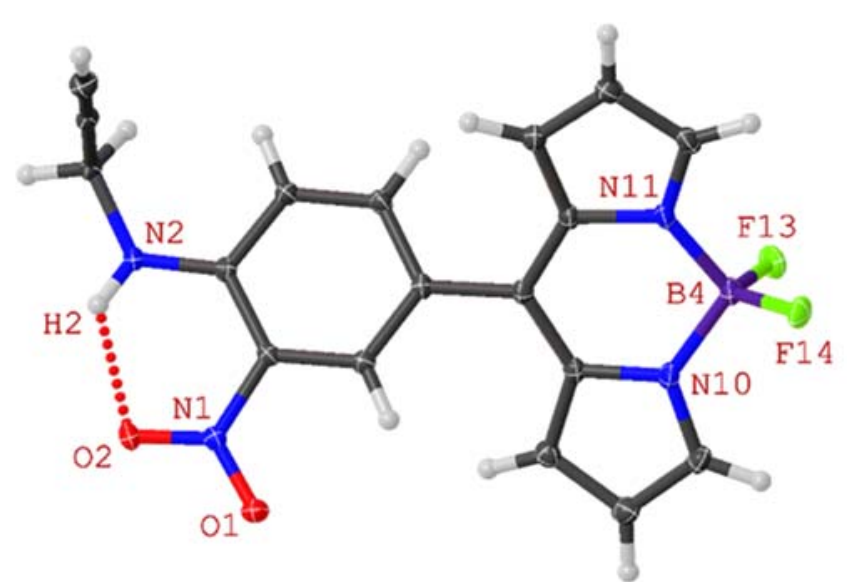

Figure 1 Molecular structure of 11a showing the intramolecular interaction between $\mathrm{N}(2) \cdots \mathrm{O}(2)\left(2.6256(11) \AA ̊ \mathrm{~N} \cdots \mathrm{O}, 129.9(12)^{\circ}\right)$. 


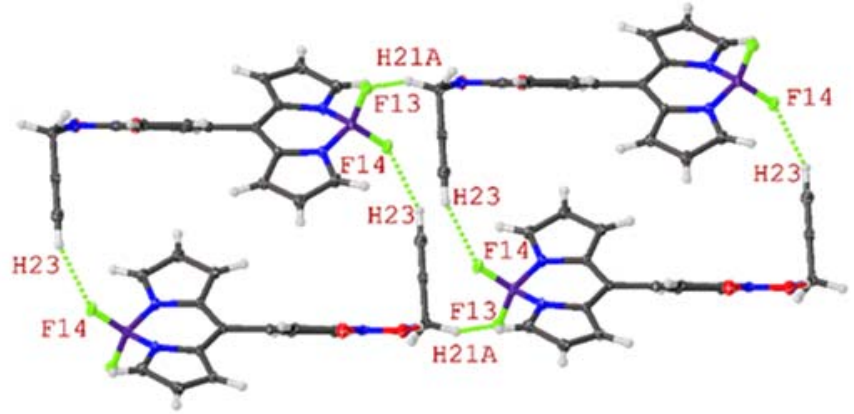

Figure 2 Expanded structure of 11a showing the head-to-tail linear network $\{C(21) \cdots F(13)$ $\left.\left[2.9835(11) \AA \mathrm{C} \cdots \mathrm{F}, 126.2(7)^{\circ}\right]\right\}$ and the head-to-tail stacked patterns $\{C(23) \cdots F(14)$ $\left.\left.3.2348(12) \AA ̊ \cap \cdots F, 156.9(2)^{\circ}\right]\right\}$.

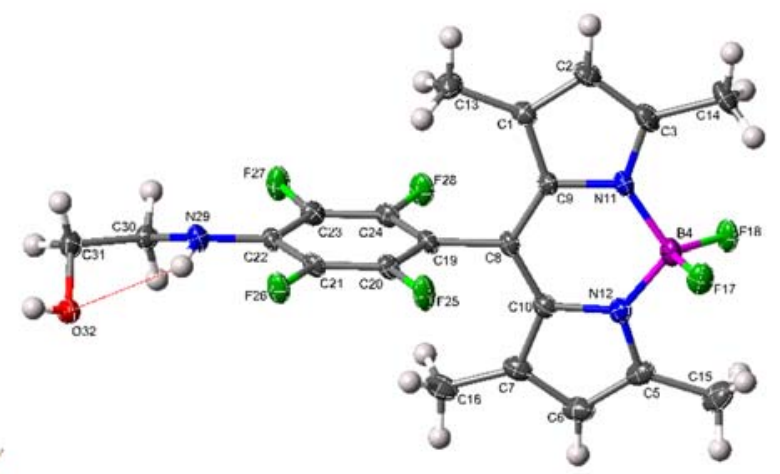

Figure $\mathbf{3}$ Molecular structure of $\mathbf{2 9}$ showing the intramolecular hydrogen bonding in the ethanolamino-moiety $\left(2.736(3) \AA ̊ N \cdots 0,111(2)^{\circ}\right)$

Intramolecular $\mathrm{N}(29)-\mathrm{H}(29) \cdots \mathrm{O}(32)$ hydrogen-bonding interactions are observed $\left(2.736(3) \AA \AA N \cdots O, 111(2)^{\circ}\right)$ in addition to intermolecular $\mathrm{O}(32)-\mathrm{H}(32) \cdots \mathrm{F}(17)$ hydrogen-bonding to adjacent molecules $\left(2.799(3) \AA ̊ 0 . \cdots, 171(4)^{\circ}\right)$. Intramolecular interactions arrange these molecules into hydrogen-bonded head-to-tail supramolecular chains, in two directions parallel to the crystallographic $b c$ - plane; one of these supramolecular chains is shown in Figure S4. A unit cell packing diagram of $\mathbf{2 9}$ is shown in Figure S5 (see Supporting Information.

Finally, halogenation with the functionalized tetramethyl-BODIPYs was investigated (Table 4). The targeted substitution of the 2- and 6position with bromine was expected to be significantly improved due to blocking other possible substitution sites of the BODIPY core structure. The targeted halogenation of 1,3,5,7-tetramethyl BODIPYs has been described in the literature by dissolving the BODIPY in an appropriate solvent and treating it with NBS or bromine..$^{25,31 b, 33}$ Given the short reaction times observed with the simple BODIPYs and NBS in HFIP (see above, Scheme 1) we decided for these reaction conditions for the 1,3,5,7-tetramethyl-substituted BODIPYs. The prefunctionalized BODIPYs (25 - 33) were dissolved in HFIP and reacted with NBS (Table 4). Controlling the reaction process by TLC, showed a very fast, nearly instantaneous, formation of the product. Therefore, the reaction time was reduced to $1 \mathrm{~min}$. In all cases, the desired 2,6-dibromo-1,3,5,7-tetramethyl-BODIPYs were formed as expected and could easily be purified by chromatography. The BODIPYs were obtained in moderate to good yields between 44 63\% (Table 4).
3

28

36

4

29

37

Table 4 Halogenation of BODIPYs 25 - 33 with NBS.

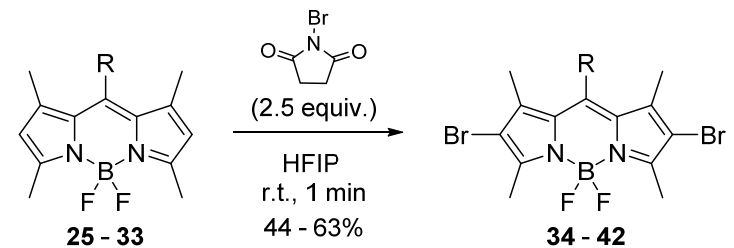

\begin{tabular}{lllll} 
Entry & $\begin{array}{l}\text { Starting } \\
\text { Material }\end{array}$ & Substituent (R) & Product & Yield [\%] \\
\hline 1 & $\mathbf{2 5}$ & & $\mathbf{3 4}$ & 57
\end{tabular}

$6 \quad 30$

39

7

31

40

44

8

32

41

54

9

33

42

48

The BODIPYs 35 and 39, which had been pre-functionalized with an $n$-butylamino group (Table 4 ) provided as well high yields. Good yields were also observed with BODIPYs carrying additional hydroxy groups (Table 4, entry 4, 8, and 9). Pre-functionalized BODIPYs, with the prop-2-ynyl moiety, were as well successfully substituted at their 2- and 6-positions in moderate to good yields (Table 4, entry 3 and 7). Thus, the synthesis of the desired 2,6-dibromo-BODIPYs could be significantly improved using the 1,3,5,7-tetramethyl-substituted BODIPYs.

Carbohydrates are important synthons when it comes to increase the biological activity of therapeutically interesting molecules. ${ }^{34}$ The 
increased number of hydroxy groups has a significant influence on the solubility in polar solvents. In addition to this, the introduction of carbohydrates is a straightforward method to increase the bioavailability of therapeutic compounds. The solubility and the cellular uptake of peptides and antitumor agents can be significantly improved by connecting them to carbohydrates. ${ }^{35}$ Also for BODIPYs, there are a few examples for coupling with carbohydrates, e.g., via extended linking groups. ${ }^{36}$ Therefore, it seems attractive to amend the series of BODIPYs with compounds carrying sugar moieties. A fast and effective method for direct glycosylation is the reaction with thio-carbohydrates, via $\mathrm{S}_{\mathrm{N}} \mathrm{Ar}$, which has successfully been applied to porphyrins and corroles. ${ }^{28,37}$ This glycosylation can often be done with the unprotected thio-carbohydrates, as shown for porphyrinoids and metal complexes. ${ }^{28 a}, 38$ Very recently, the synthesis of two glyco-substituted BODIPYs has been described using acetoxyprotected thio-carbohydrates starting with glycosylation of the dipyrromethane. ${ }^{39}$ In analogy to the literature on porphyrinoids, ${ }^{28 a, 38 a}$ we decided instead to test the glycosylation with unprotected thio-carbohydrates directly on the tetramethyl BODIPYs 25 and 26. For this, BODIPY 25 was dissolved with the corresponding 1 '-thio- $\beta$-D-carbohydrate sodium salt (glucose or galactose) in DMF and was reacted at room temperature (Scheme 3). Within $15 \mathrm{~min}$ reaction time the reaction was complete and the corresponding glycosylated BODIPYs were obtained. The glucosyl conjugate $\mathbf{4 3}$ was isolated in $69 \%$ and the galactosyl conjugate $\mathbf{4 4}$ in $46 \%$ yield. The glycosylated BODIPYs $\mathbf{4 3}$ and $\mathbf{4 4}$ were then subjected to halogenation with NBS in HFIP. Also, for these complex molecules, the corresponding 2,6-dibromo-substituted BODIPYs were readily obtained (45 and $\mathbf{4 6}$ ) within a reaction time of one minute in $62 \%$ and $58 \%$ yield, respectively (Scheme 3). Analogously, BODIPY 26 was treated with the respective $1^{\prime}$-thio- $\beta$-D-carbohydrate sodium salts, yielding to the glycosylated BODIPYs 47 and $\mathbf{4 8}$ followed by bromination, resulting in the dibrominated and glycosyl-substituted BODIPYs 49 and $\mathbf{5 0}$ (Scheme 4). This simple post-functionalization with unprotected thio-carbohydrates could also be extended to the direct glycosylation of BODIPYs $\mathbf{1 a}$ and $\mathbf{8 a}$, carrying the pentafluorophenyl and the 4-fluoro-5-nitrophenyl substituent, respectively (Scheme 5). The glucosyl- and galactosyl-substituted BODIPYs $\mathbf{5 1} \mathbf{-} \mathbf{5 4}$ were obtained in very high to excellent yields (Scheme 5). The BODIPY-glycoconjugates $\mathbf{5 1} \mathbf{-} \mathbf{5 4}$ served as nonhalogenated comparators in the subsequent bacterial assays (see below) with BODIPY conjugates $\mathbf{4 3}$ - 50, which combine the carbohydrate substituent and the 1,3,5,7-tetramethyl/2,6-dibromo substitution pattern.

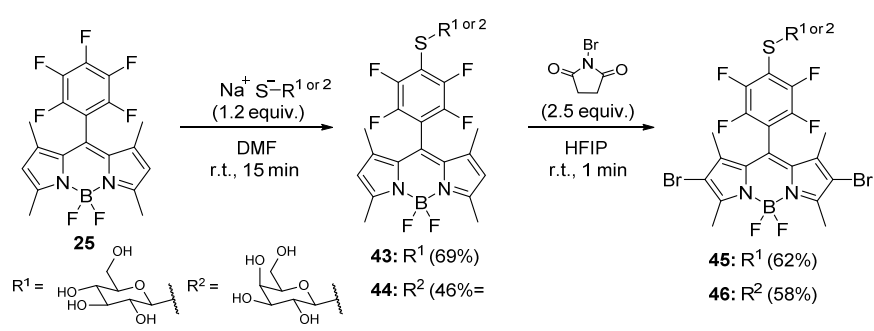

Scheme 3 Glycosylation of BODIPY 25 and halogenation with NBS.
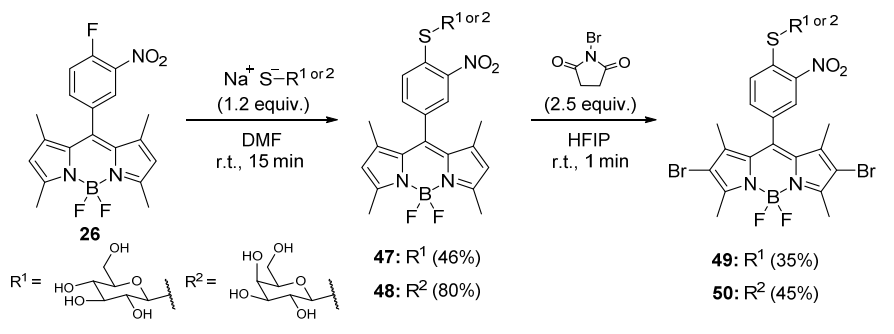

49: $\mathrm{R}^{1}(35 \%)$

50: $R^{2}(45 \%)$

Scheme $\mathbf{4}$ Glycosylation of BODIPY 26 and halogenation with NBS.

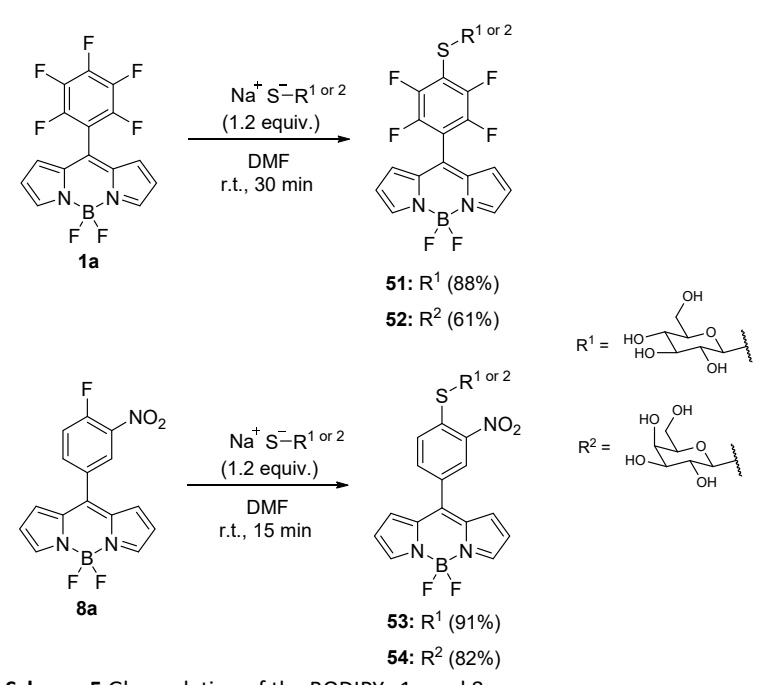

Scheme 5 Glycosylation of the BODIPYs $1 \mathrm{a}$ and $8 \mathrm{a}$.

\section{Evaluation in assays against $S$. aureus}

For studying their antimicrobial properties, the synthesized BODIPYs were tested in bacterial assays against $S$. aureus with and without irradiation. S. aureus is an attractive target because it is a typical member of the microflora of wounds with a high tendency to develop antibiotic resistance. ${ }^{40}$ Results for homologous series of compounds are presented in Figure 4-9, additionally; results for a selection of monobrominated BODIPYs are given in Figure 10 (bacterial tests for the other synthesized BODIPYs can be found in the Supporting Information). For a possible antimicrobial application in clinical practice the dyes need to be active in the presence of body fluids in complex biological environments. In blood serum or (burn) wounds typically different types of proteins are present. These protein-rich environments can significantly reduce the effectiveness of photosensitizers. ${ }^{22,41}$ Therefore, in addition to tests in PBS further investigations were conducted in PBS with the addition of horse serum $(10 \%)$ to more realistically model the environment where the BODIPYs would need to exert photoactivity. As mentioned above, the investigations also included BODIPYs with a non-functionalized core structure (no methyl groups, no bromine substitution) for comparison to the modified BODIPYs. Typically, these nonfunctionalized BODIPYs are expected to show only a limited or no phototoxic activity. For the bacterial assays, cultures of $S$. aureus were tested under different conditions (incubation with three BODIPY concentrations, 1, 10, and $100 \mu \mathrm{M}$, with and without addition of serum followed by light exposure; no BODIPY and absence of light; and $100 \mu \mathrm{M}$ of the BODIPY - the highest concentration - in the absence of light to identify dark toxicity). For 
investigation of the phototoxicity, the bacteria were incubated with the corresponding BODIPY (in concentrations of 1, 10, and $100 \mu \mathrm{M}$ ) for 30 min in PBS and in PBS with $10 \%$ horse serum. The samples were then exposed to white light with a power density and irradiation time resulting in an energy fluence of about $100 \mathrm{~J} / \mathrm{cm}^{2}$. The short incubation time of $30 \mathrm{~min}$ - short in comparison to investigations of PDT against tumor cells, where often an incubation time of $24 \mathrm{~h}$ is used - was chosen with respect to the specific recommendations of antibacterial therapy; bacteria reproduce much more rapidly than cells, hence, activity is needed after shorter residence times. ${ }^{42}$ Bacterial inactivation is presented as the logarithm of the number of colony-forming units, Ig (CFU $\mathrm{mL}^{-1}$ ), taking into account that a reduction of several log stages is usually required for a successful antibacterial treatment. As a control experiment the effect of light only on the bacterial suspensions was also tested (see Supporting Information; Figure S15), which did not exert a measurable effect.

Interestingly, in contrast to what may be expected, some of the paraamino-substituted BODIPYs with a non-functionalized core structure exhibited a strong phototoxicity in PBS, where a complete inactivation of the bacteria was observed already at $1 \mu \mathrm{M}$ (Figure 4, 3a, 4a, 9a, and 11a), with no inactivation of bacteria occurring in the absence of light. Of the glucosyl-substituted BODIPYs $\mathbf{5 1}$ and $\mathbf{5 3}$ (with a non-functionalized core structure), the tetrafluorophenylsubstituted BODIPY $\mathbf{5 1}$ showed an efficient phototoxic activity at the highest concentration against $S$. aureus in PBS (Figure 4), whereas the 3-nitrophenyl-substituted compound $\mathbf{5 3}$ (Figure 4, right side) only exhibited a small phototoxic effect at $100 \mu \mathrm{M}$ (the galactosyl- substituted compound $\mathbf{5 2}$ showed only a small phototoxic effect at $100 \mu \mathrm{M}$ and $10 \mu \mathrm{M}$, while $\mathbf{5 4}$ exhibited no significant activity, see Supporting Information; Figure S6). Also, in subsequent investigations there is a tendency for the tetrafluorophenylsubstituted BODIPYs to have a higher bactericidal activity than the corresponding 3-nitrophenyl-substituted compounds (see Figures 5 -9 , below). In contrast to the results in PBS, the inactivation of $S$. aureus by the BODIPYs $3 a, 4 a, 9 a$, and $11 a$ and the others drastically decreased, and in some cases vanished, in the presence of $10 \%$ of serum (Figure 5). Here, no efficient inactivation of the bacteria was observed, although for some compounds, $\mathbf{4 a}, \mathbf{9 a}$, and $\mathbf{1 1 a}$, at least a one stage reduction (90\%) of bacterial viability was found. From the comparison of Figure 4 and Figure 5 it becomes apparent how strongly the addition of serum influences the antibacterial activity, suggesting that the use of the PBS-serum combination as a more realistic model can indeed help to screen and identify candidates suitable for further aPDT testing. ${ }^{22,41}$ Figure 6 shows the results in PBS for the series of BODIPYs substituted with methyl groups at their 1, 3, 5, and 7-positions. Compared to the non-methyl-substituted congeners the antibacterial activity seems diminished in this series of BODIPYs. Nevertheless, for BODIPYs 29, 32, and 43 a complete inactivation of $S$. aureus at least at the highest concentration is observed. Only the two BODIPYs carrying the $n$-butylamino group (27 and 30) exhibit a strong activity with complete inactivation of $S$. aureus already at a concentration of $10 \mu \mathrm{M}$ (Figure 6). BODIPYs 28, 31, and $\mathbf{4 7}$ show a medium activity with a three-log stage reduction of bacterial viability.

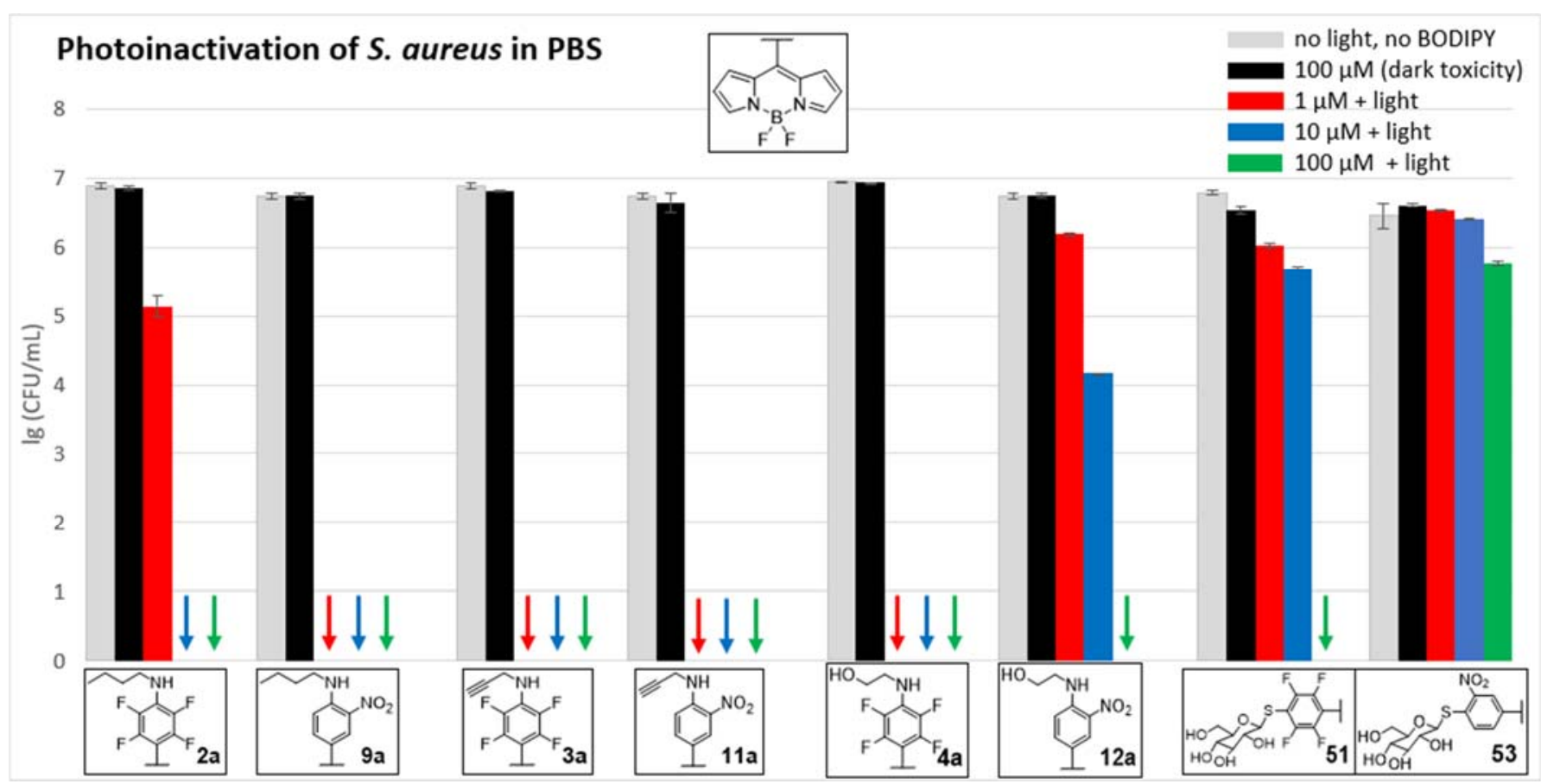

Figure 4 Photoinactivation of $S$. aureus by selected BODIPYs with a non-functionalized core structure ( 30 min incubation and irradiation with white light) in phosphate-buffered saline (PBS). The antibacterial toxicity is expressed as logarithm of the number of colony-forming units, $\lg \left(\mathrm{CFU} \mathrm{mL}^{-1}\right.$ ). " $\downarrow$ " indicates suppression of bacterial growth below the detection limit. 


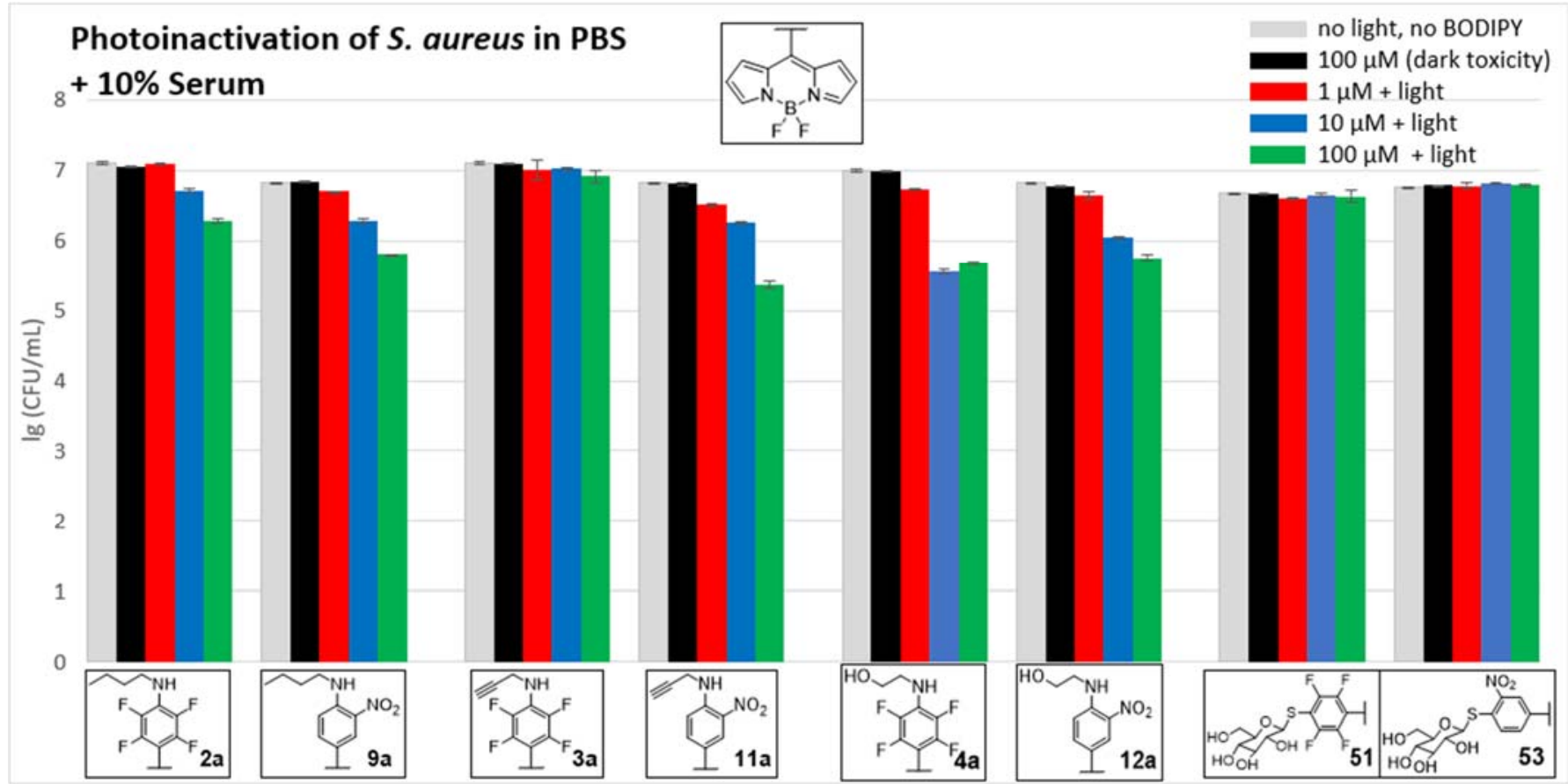

Figure 5 Photoinactivation of $S$. aureus by selected BODIPYs with a non-functionalized core structure ( 30 min incubation and irradiation with white light) in phosphate-buffered saline (PBS) with $10 \%$ serum. The antibacterial toxicity is expressed as logarithm of the number of colony-forming units, lg (CFU $\left.\mathrm{mL}^{-1}\right)$.

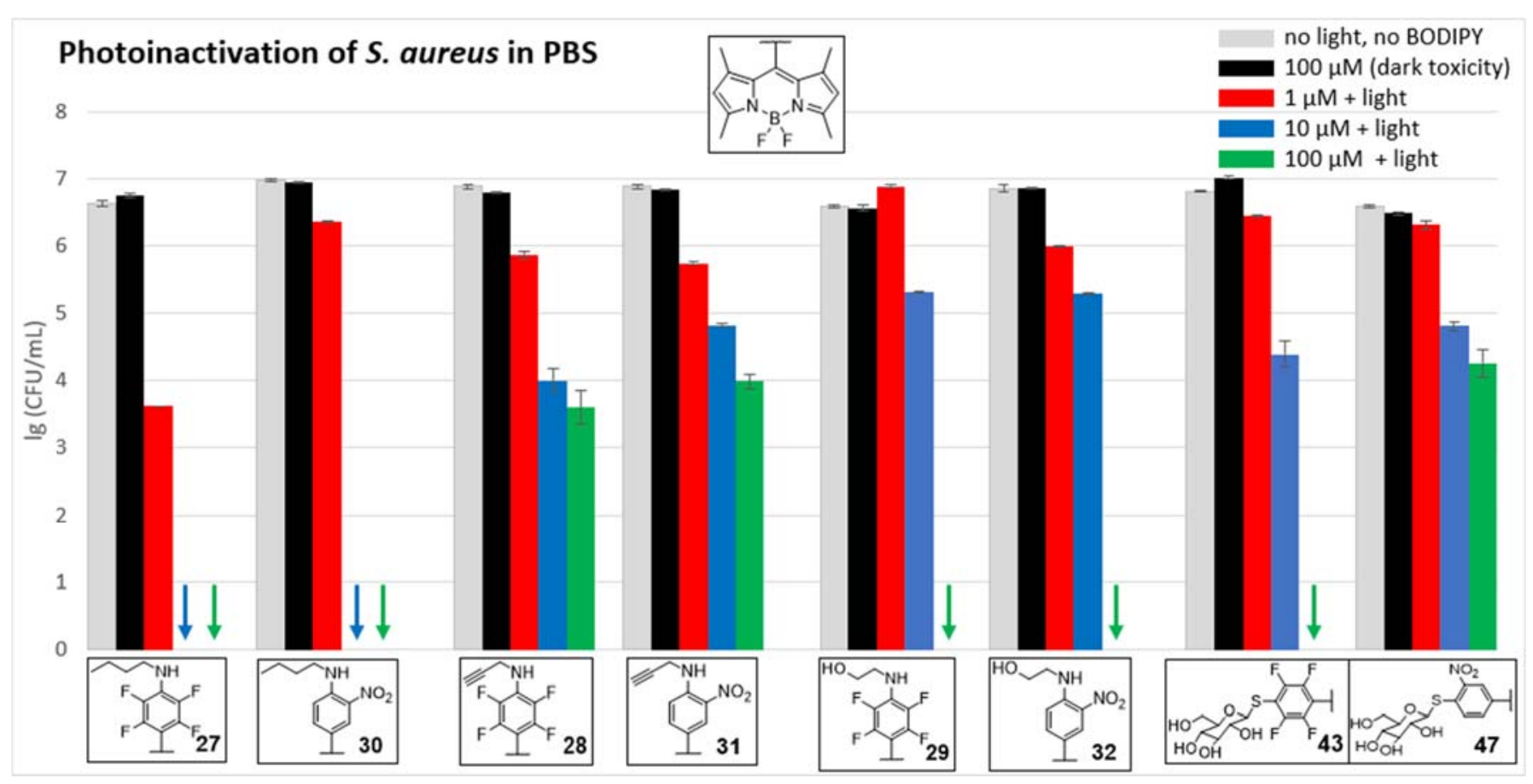

Figure 6 Photoinactivation of S. aureus by selected 1,3,5,7-tetramethyl substituted BODIPYs (30 min incubation and irradiation with white light) in phosphate-buffered saline (PBS). The antibacterial toxicity is expressed as logarithm of the number of colony-forming units, lg (CFU mL $\mathrm{L}^{-1}$ ). “ $\downarrow$ ” indicates suppression of bacterial growth below the detection limit. 


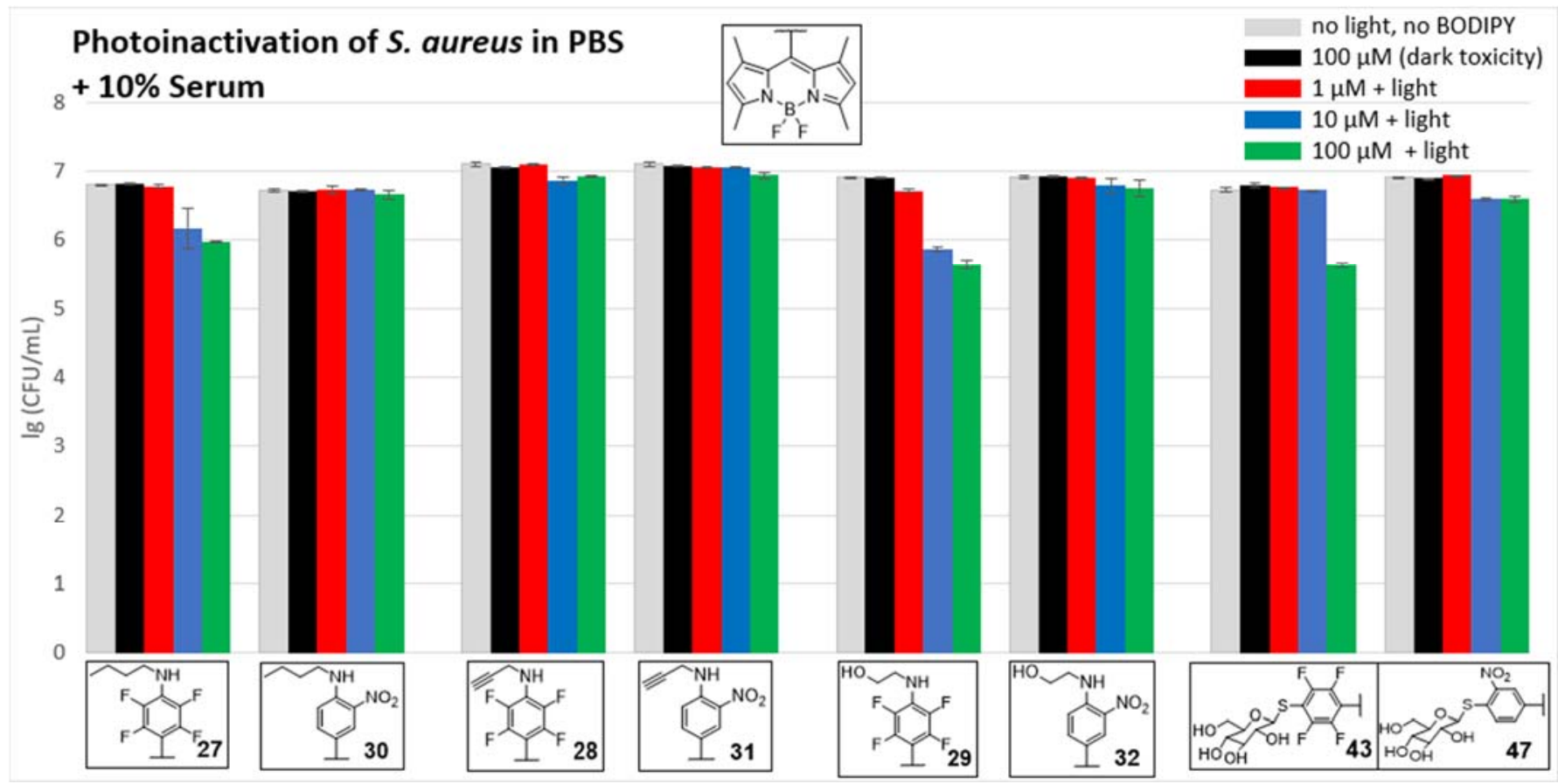

Figure 7 Photoinactivation of S. aureus by selected 1,3,5,7-tetramethyl substituted BODIPYs (30 min incubation and irradiation with white light) in phosphate-buffered saline (PBS) with $10 \%$ serum. The antibacterial toxicity is expressed as logarithm of the number of colony-forming units, $\lg \left(\mathrm{CFU} \mathrm{mL} \mathrm{mL}^{-1}\right)$.

On repeating the test of the tetramethyl-substituted BODIPYs with the addition of serum (Table 7), again no efficient inactivation of the bacteria could be observed, though here again a small reduction of bacterial viability by at least one log stage $(90 \%)$ was found for $\mathbf{2 7}$, 29, and 43. Finally, the 2,6-dibromo-substituted BODIPYs were investigated in the assay against $S$. aureus. The testing of these BODIPYs - substituted with methyl groups and bromine - showed a drastic improvement of their antibacterial toxicity (Figure 8). All BODIPYs, whether para-amino substituted $(35-37,39-41)$ or thioglycosylated (45 and 49; for the galactosyl-substituted BODIPYs 46 and 50, see Supporting Information; Figure S8), exhibited a highly effective inactivation of $S$. aureus in PBS with a complete inactivation of bacteria already in a concentration of $1 \mu \mathrm{M}$ under light. However, except for BODIPYs 35, $\mathbf{3 9}$ and the galactosyl-substituted BODIPY $\mathbf{5 0}$ (see Supporting Information; Figure S8), all BODIPYs showed also a strong dark toxicity at the highest concentration $(100 \mu \mathrm{M})$ of the BODIPY, resulting in a complete inactivation of the bacteria (Figure 8 , black arrows). Having these highly active BODIPYs at hand they were now again challenged with the antibacterial tests being performed in the presence of $10 \%$ of serum. Not unexpectedly as for the previous compounds a significant decrease in their activity was observed under these conditions. Several of the tested BODIPYs showed no significant phototoxicity and no dark toxicity anymore (35 - 37, and 39). However, with these methyl- and bromo-substituted BODIPYs, for the first time in the series of experiments a complete inactivation of bacteria in the presence of serum is found for some compounds, namely BODIPY $\mathbf{4 0}$ and the ethylamino- and thioglycosyl-substituted BODIPYs (Figure 9). Specifically, the glycosylated conjugates (45 and 49; for 46 and 50 see again Supporting Information; Figure S13) exhibited a similar influence on the bacteria in the presence of serum compared to the results in PBS (Figure 8). For BODIPY $\mathbf{4 5}$ there is no difference in the inactivation of the bacteria in PBS and in PBS plus serum, while $\mathbf{4 9}$ still provided a dominant phototoxic activity at the two higher concentrations of the BODIPY (Figure 9; the same holds for the galactosyl-substituted compound 46, whereas the galactosyl-substituted BODIPY $\mathbf{5 0}$ exhibited a complete inactivation of bacteria even at $1 \mu \mathrm{M}$, see Supporting Information; Figure S13).

Having the monobrominated BODIPYs at hand (see above Table 2) their antibacterial activity was also investigated. These monobrominated BODIPYs $(\mathbf{1 9}, \mathbf{2 0}, \mathbf{2 3}$, and24) showed a significant inactivation of the bacteria in PBS, similar to the BODIPYs with an unmodified core structure (see Supporting Information; Figure S10). BODIPY 23 and 24 exhibited dark toxicity at $100 \mu \mathrm{M}$ concentration in PBS, while, the BODIPYs $\mathbf{1 9}$ and $\mathbf{2 0}$ showed no significant differences in their antimicrobial activity in PBS (see Supporting Information; Figure S10). Interestingly, the tested monobrominated BODIPYS (19, 20, 23, and 24) also exhibited a significant inactivation of $S$. aureus in the presence of serum (Figure 10). In all cases, a complete inactivation of the bacteria was observed at the highest concentration of the BODIPY. Of those, BODIPY $\mathbf{2 4}$ still showed a dominant toxicity in the absence of light. In comparison to the highly active dibromo-substituted BODIPYs (45 and 49; 46 and 50, see Supporting Information; Figure S13), the monobrominated conjugates 19, 20, 23, and 24 showed a lower antimicrobial activity; however, these monobrominated BODIPYs still had a notable effect on $S$. aureus in the presence of serum. This is specifically remarkable as they do not contain sugar moieties, like the most effective dibromo-substituted compounds. 


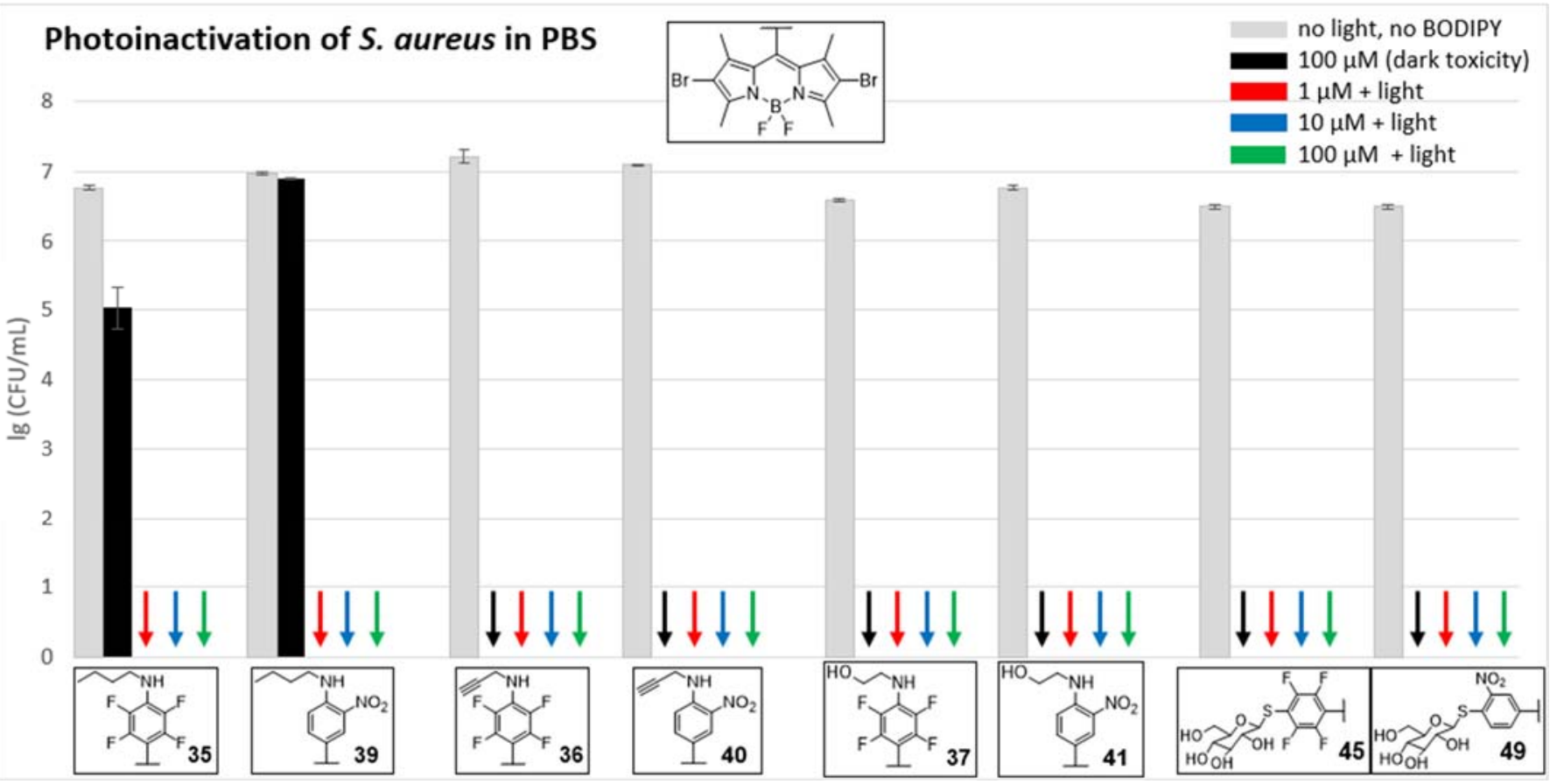

Figure 8 Photoinactivation of S. aureus by selected 2,6-dibromo-substituted BODIPYs ( 30 min incubation and irradiation with white light) in phosphate-buffered saline (PBS). The antibacterial toxicity is expressed as logarithm of the number of colony-forming units, lg (CFU mL-1). " $\downarrow$ " indicates suppression of bacterial growth below the detection limit.

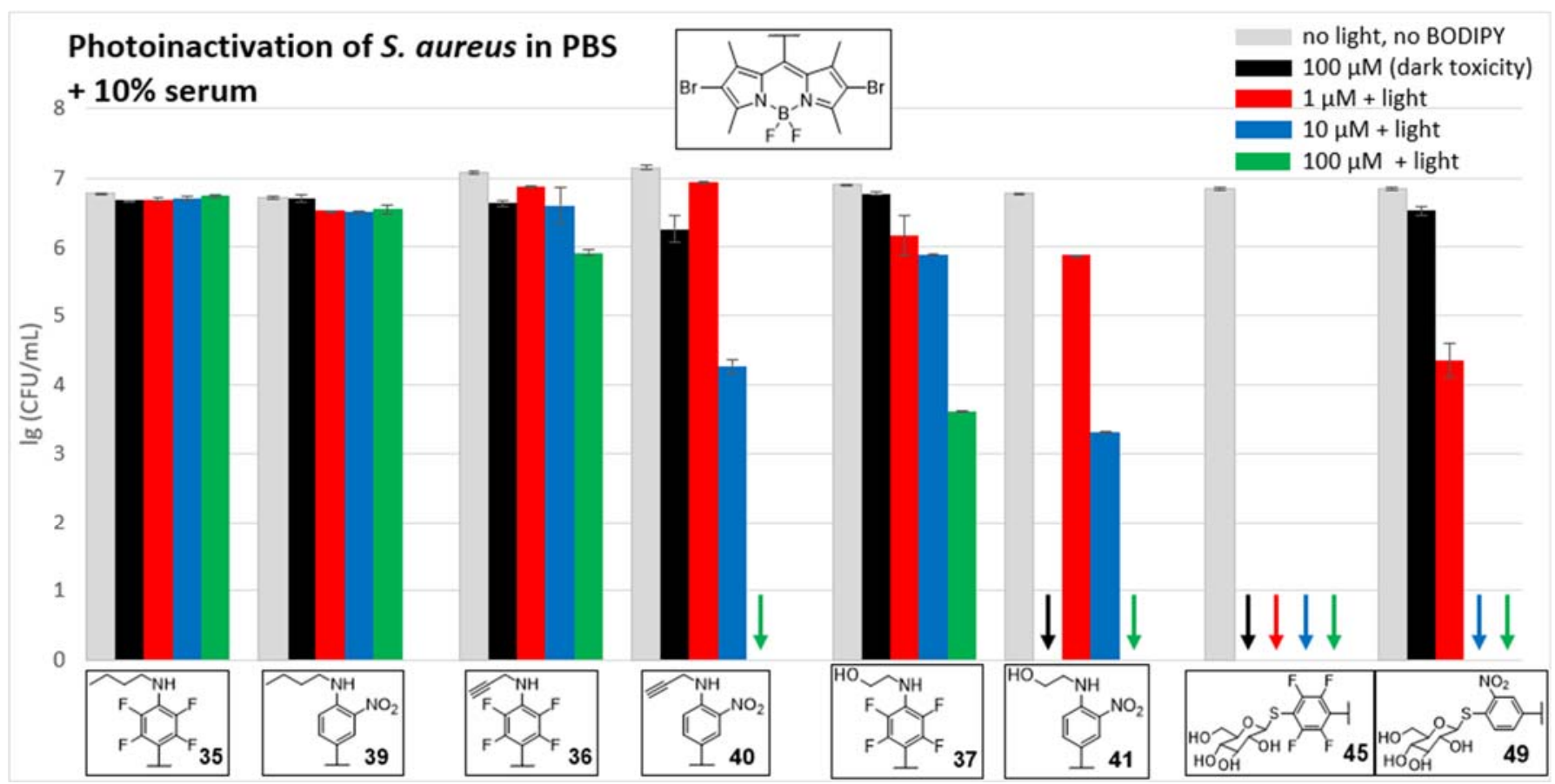

Figure 9 Photoinactivation of S. aureus by 2,6-dibromo-substituted BODIPYs (30 min incubation and irradiation with white light) in phosphate-buffered saline (PBS) with 10\% serum. The antibacterial toxicity is expressed as logarithm of the number of colony-forming units, lg (CFU mL-1). “ $\downarrow$ ” indicates suppression of bacterial growth below the detection limit. 


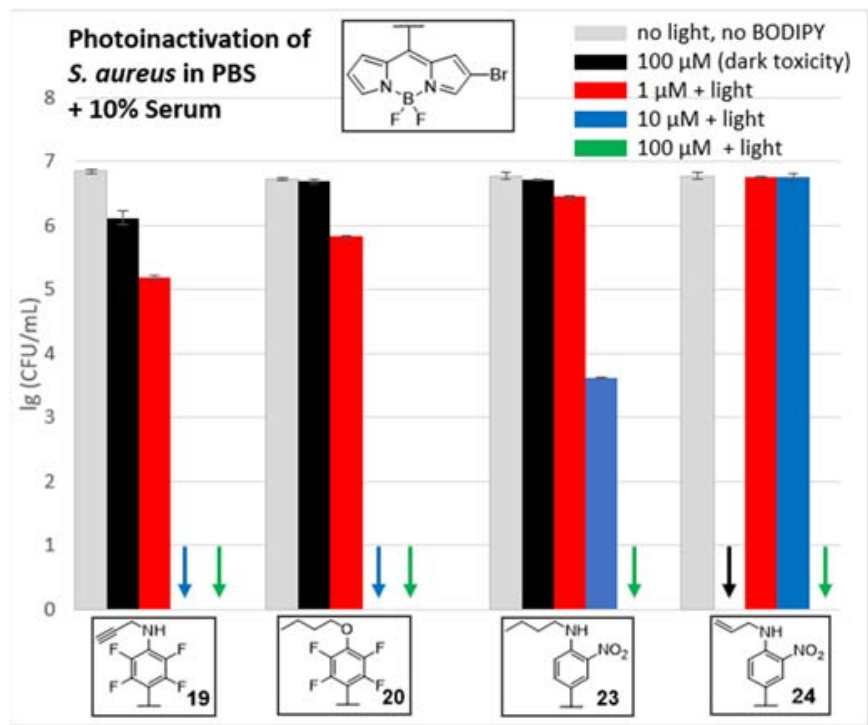

Figure 10 Photoinactivation of $S$. aureus by selected 2-bromo-substituted BODIPYs (30 min incubation and irradiation with white light) in phosphate-buffered saline (PBS) with $10 \%$ serum. The antibacterial toxicity is expressed as logarithm of the number of colony-forming units, Ig (CFU mL ${ }^{-1}$ ). “ $\downarrow$ " indicates suppression of bacterial growth below the detection limit

Their high antibacterial activity may be due to the specific effect of monobromination of BODIPYs on the photophysical properties relevant for aPDT. ${ }^{43}$

In a last set of experiments the dibrominated BODIPYs were tested against the Gram-negative germ $P$. aeruginosa (Figures 11 and 12). $P$. aeruginosa has a high tendency to develop antibiotic resistance and is a major threat in nosocomial infections..$^{44,45,46}$ It has also been the target in several aPDT investigations. ${ }^{47}$

The tests were conducted in the same way as for $S$. aureus: incubation with three BODIPY concentrations, 1, 10, and $100 \mu \mathrm{M}$, with and without addition of serum followed by light exposure; no
BODIPY and absence of light; and $100 \mu \mathrm{M}$ of the BODIPY - the highest concentration - in the absence of light to identify dark toxicity. The 'light only' experiment was performed with $P$. aeruginosa as well (for all details see Supporting Information; Figure S15).

Figure 11 shows the results from the tests with the dibrominated BODIPYs against $P$. aeruginosa. Not unexpectedly, the activity of the dibrominated BODIPYs is significantly lower than against $S$. aureus. A reduction of bacterial viability of about $1.5 \mathrm{log}$ stages is achieved with BODIPYs 35, 37, 40 in the highest concentration. The best antibacterial activity, however, is again exhibited by the glycosylated derivatives $\mathbf{4 5}, \mathbf{4 6}$, and $\mathbf{4 9}$. For $\mathbf{4 5}$ and $\mathbf{4 6}$ a reduction of bacterial viability by 3 log stages is observed in the highest concentration of $100 \mu \mathrm{M}$. This is a remarkable activity against a Gram-negative bacterium for a non-charged photosensitizer, as typically these antibacterial photosensitizers are charged (cationic) compounds, like methylene blue. ${ }^{4 c, 47 a, c}$ Those cationic photosensitizers, however, bear the risk of unwanted DNA interaction with mutagenic potential. ${ }^{4 c, 48}$ BODIPYs $\mathbf{4 5}$ and $\mathbf{4 6}$ are also the only ones which exert an antibacterial activity against $P$. aeruginosa even in PBS supplemented with $10 \%$ of serum (Figure 12). Here, $85 \%$ and $88 \%$ reduction of bacterial viability, for 45 and $\mathbf{4 6}$, respectively, is observed. Interestingly, for $P$. aeruginosa, again the different effect of the 2,3,5,6-tetrafluorophenyl vs. the 3-nitrophenyl-substitution is found. The 4-glycosyl-2,3,5,6-tetrafluorophenyl-substituted compounds exert a higher antibacterial activity. Though this antibacterial activity of the BODIPYs against Gram-negative $P$. aeruginosa is still limited the bromination/glycosylation strategy presents itself as a promising approach for photoactive antibacterial compounds.

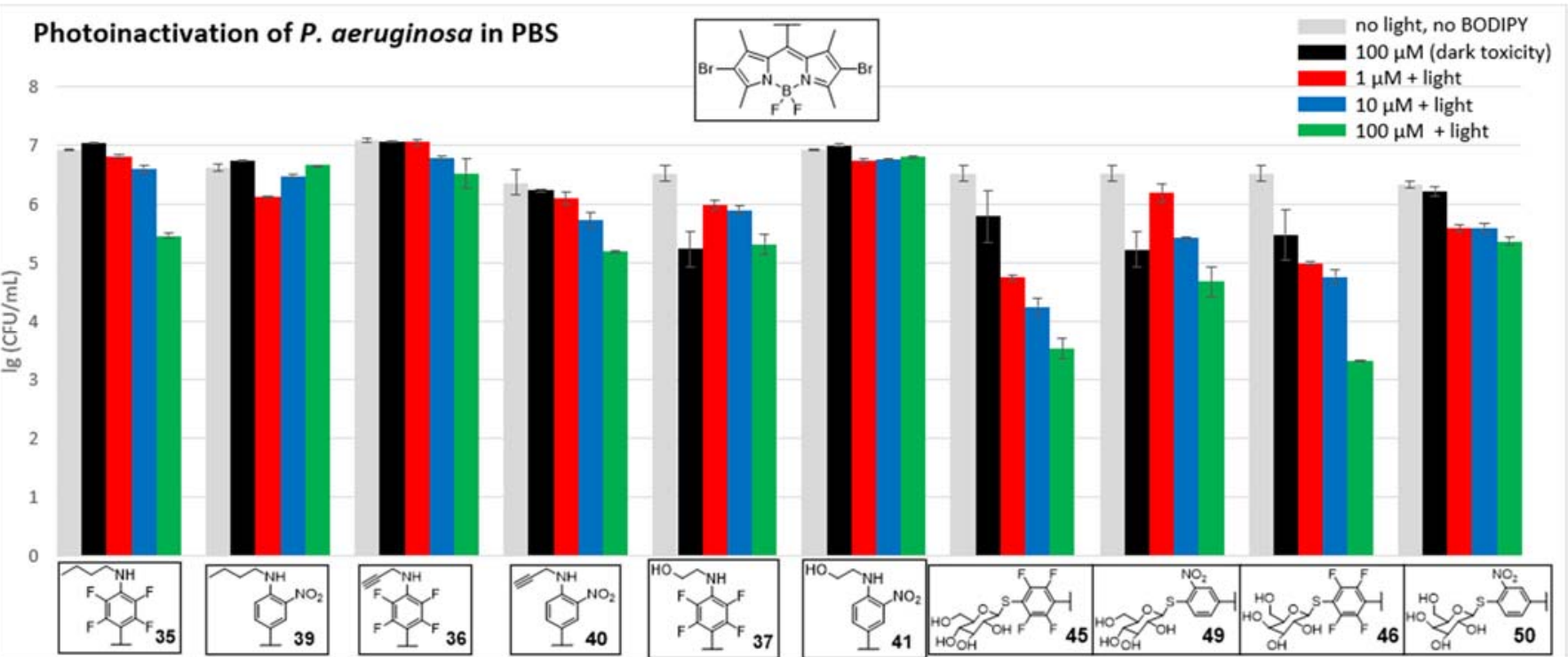

Figure 11 Photoinactivation of $P$. aeruginosa by selected 2,6-dibromo-substituted BODIPYs (30 min incubation and irradiation with white light) in phosphate-buffered saline (PBS). The antibacterial toxicity is expressed as logarithm of the number of colony-forming units, Ig (CFU $\mathrm{mL}^{-1}$ ). 


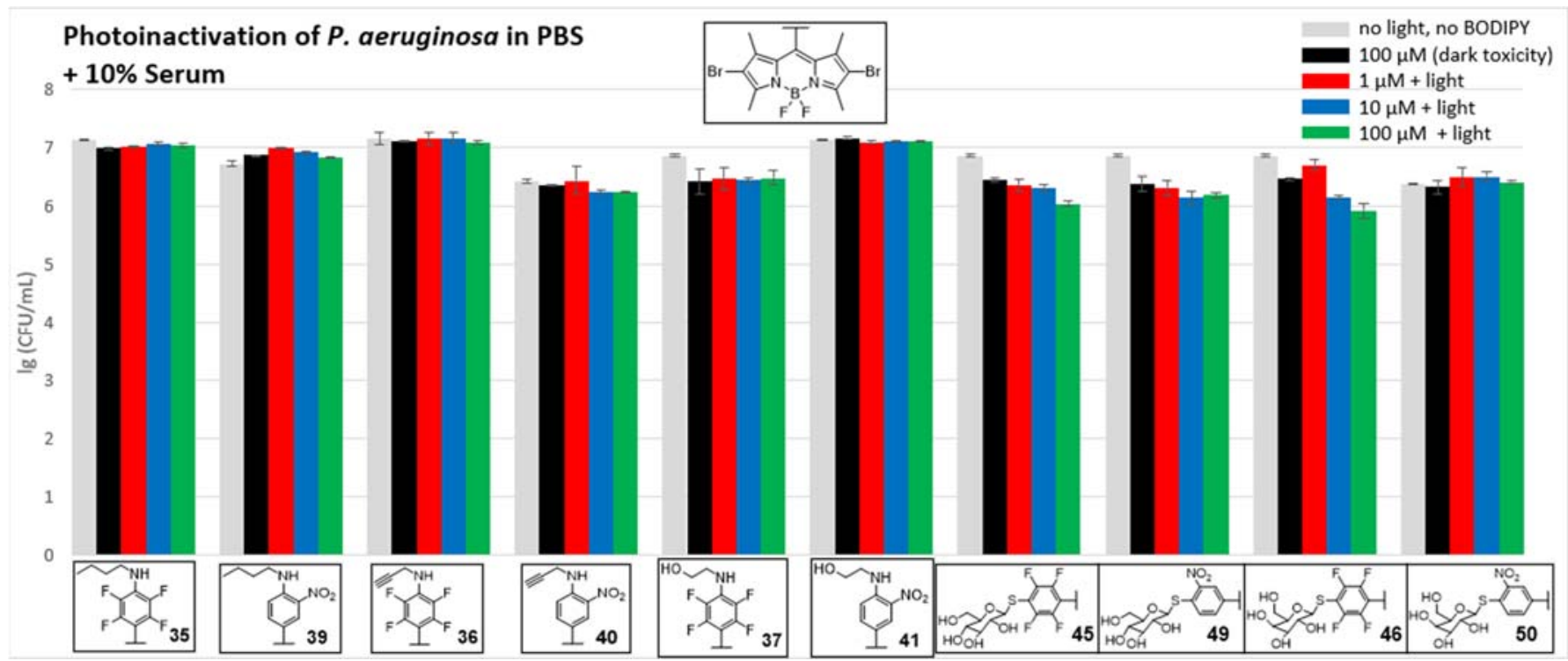

Figure 12 Photoinactivation of $P$. aeruginosa by 2,6-dibromo-substituted BODIPYs (30 min incubation and irradiation with white light) in phosphate-buffered saline (PBS) with $10 \%$ serum. The antibacterial toxicity is expressed as logarithm of the number of colony-forming units, $\lg$ (CFU mL-1).

\section{Summary and conclusions}

In this work the stepwise optimization of the 8-pentafluorophenyland the 8-(4-fluoro-3-nitrophenyl)-BODIPY for a possible application in aPDT was investigated. These basic BODIPY structures were chosen because they can easily be modified at their para-position with a multitude of nucleophiles. As halogenation is known to influence the photophysical properties of BODIPYs in a way favorable for aPDT, first a targeted bromination of the BODIPY core structure was investigated. Pre-functionalized BODIPYs with a nonfunctionalized core structure were successfully monobrominated. However, selective dibromination proved to be difficult and often led to inseparable BODIPY mixtures. Therefore, the respective 1,3,5,7tetramethyl substituted BODIPYs, carrying the pentafluorophenyl and the 4-fluoro-3-nitrophenyl moiety in their meso-positions were synthesized. These BODIPYs were substituted with several amines via $\mathrm{S}_{\mathrm{N}} \mathrm{Ar}$. Also, a direct glycosylation was successfully achieved via a simple nucleophilic substitution with unprotected thiocarbohydrates. Subsequently, the various substituted tetramethyl BODIPYs were selectively dibrominated via halogenation with NBS. With this synthetic strategy four series of BODIPYs were obtained which were then evaluated for their antibacterial activity against $S$. aureus with and without irradiation. Tests were first performed in PBS but then also with the addition of serum to more realistically model the complex biological environment relevant for bacterial infections. Many of the tested BODIPYs exhibited a strong antibacterial activity against $S$. aureus in PBS, sometimes also an antibacterial activity even in the absence of light. However, in many cases this antibacterial activity greatly decreased or vanished completely in the presence of serum. Nevertheless, some BODIPYs still showed a pronounced antimicrobial activity on the bacteria in the presence of serum. A very effective inactivation - even in the presence of serum - was observed with the 2,6-dibromo-1,3,5,7tetramethyl substituted BODIPYs, carrying a thio-carbohydrate group. Interestingly, some simple monobrominated BODIPYS, without carbohydrate substitution, also exhibited a significant inactivation of $S$. aureus in the presence of serum. Finally, the dibrominated BODIPYs were tested in the inactivation of the Gramnegative bacterium $P$. aeruginosa. It turned out that the glycosylated, dibrominated BODIPYs were able to reduce bacterial viability by 3 log stages suggesting that such glycosylated, dibrominated BODIPYs could be promising candidates for aPDT.

\section{Experimental}

\section{General remarks}

All reactions were performed in standard round bottom flasks. DCM, $n$-pentane, and methanol were purchased and used as received. Other solvents were purchased and distilled at reduced pressure. Purchased chemicals were used as received without further purification. All liquid reagents were added through syringes. Reactions were monitored by thin-layer chromatography (Merck, TLC Silica gel $60 \mathrm{~F}_{254}$ and visualized under UV light $(254 \mathrm{~nm}$ and 366 $\mathrm{nm})$. Flash column chromatography was performed on silica gel (Fluka silica gel 60M, 40-63 $\mu \mathrm{m}$ ). NMR spectra were recorded with JEOL ECX400, JEOL ECP500, JEOL ECZ600, and Bruker Avance700 instruments. Multiplicity of the signals was assigned as follows: $s=$ singlet, $\mathrm{br} \mathrm{s}=$ broad singlet, $\mathrm{d}=$ doublet, $\mathrm{t}=$ triplet, $\mathrm{dd}=$ doublet of doublets, $\mathrm{dt}=$ doublet of triplets, $\mathrm{tt}=$ triplet of triplets, $\mathrm{ddd}=$ doublet of doublets of doublets, $\mathrm{ddt}=$ doublet of doublets of triplets, $\mathrm{m}=$ multiplet, $\mathrm{m}_{\mathrm{c}}=$ centered multiplet. Chemical shifts are reported relative to $\mathrm{CDCl}_{3}\left({ }^{1} \mathrm{H}: \delta=7.26 \mathrm{ppm},{ }^{13} \mathrm{C}: \delta=77.2 \mathrm{ppm}\right), \mathrm{THF}-\mathrm{d}_{8}\left({ }^{1} \mathrm{H}: \delta\right.$ $\left.=3.58 \mathrm{ppm},{ }^{13} \mathrm{C}: \delta=67.6 \mathrm{ppm}\right)$, and DMSO-d $\mathrm{d}_{6}\left({ }^{1} \mathrm{H}: \delta=2.50 \mathrm{ppm},{ }^{13} \mathrm{C}:\right.$ $\delta=39.5 \mathrm{ppm})$. Coupling constants are given in hertz $(\mathrm{Hz})$. All ${ }^{13} \mathrm{C} \mathrm{NMR}$ spectra are proton-decoupled. For a detailed peak assignment 2D 
spectra were measured (COSY, HMBC, and HMQC). HRMS analyses were carried out on an Agilent Technologies 6210 ESI-TOF (electrospray ionization, time of flight) instrument. IR spectra were measured with a JASCO FT/IR 4100 spectrometer equipped with a PIKE MIRacle ${ }^{\text {TM }}$ ATR instrument. UV/Vis spectra were recorded on a SPECORD S300 UV/Vis spectrometer (Analytik Jena) in quartz cuvettes ( $1 \mathrm{~cm}$ length). The fluorescence spectra of the BODIPYs were recorded with a JASCO FP 6500 spectrofluorometer in quartz cuvettes (1 cm length). Specified melting points were recorded on a Reichert Thermovar Apparatus.

Compounds 1, 3-12, 1a, 3a, 4a, 6a-12a, 25 and 27 were prepared according to the literature $26 a, 26 b, 32$; for the synthesis of $\mathbf{2}, \mathbf{2 a}, \mathbf{5 a}$, and $\mathbf{2 6}$ see Supporting Information.

\section{General procedure for the dibromination of BODIPYs (13-16)}

The corresponding BODIPY 1a, 7a-9a (1 equiv.) was dissolved in 2 $\mathrm{mL}$ of HFIP. NBS (2.5 equiv.) was added and the mixture was stirred for the indicated time at rt. Afterwards, the reaction mixture was diluted with DCM and washed with water several times. The organic layer was dried with $\mathrm{Na}_{2} \mathrm{SO}_{4}$, filtrated and evaporated to dryness. The crude product was purified by column chromatography.

\section{General procedure for the targeted dibromination of BODIPYs} under modified conditions (13 and 15)

The corresponding BODIPY 1 a or 8 a (1 equiv.) was dissolved in DCM. NBS ( 2.5 equiv., dissolved in DCM) was added and the mixture was stirred for the indicated time at rt. Afterwards, the reaction mixture was diluted with DCM and washed with water several times. The organic layer was dried with $\mathrm{Na}_{2} \mathrm{SO}_{4}$, filtrated and evaporated to dryness. The crude product was purified by column chromatography.

\section{General procedure for the targeted monobromination of BODIPYs} (17 -24)

The corresponding BODIPY 1a, 3a, $\mathbf{5 a} \mathbf{- 1 0 a}$ (1 equiv.) was dissolved in DCM. NBS (2.5 equiv., dissolved in DCM) was added and the mixture was stirred for the indicated time at rt. Afterwards, the reaction mixture was diluted with $\mathrm{DCM}$ and washed with water several times. The organic layer was dried with $\mathrm{Na}_{2} \mathrm{SO}_{4}$, filtrated and evaporated to dryness. The crude product was purified by column chromatography.

General procedure for the nucleophilic substitution of BODIPYs with amines (28 - 33)

The corresponding BODIPY $\mathbf{2 5}$ or $\mathbf{2 6}$ ( 1 equiv.) was dissolved in DMF, DCM, or DMSO and the corresponding amine (10 - 20 equiv.) was added. The mixture was stirred for the indicated time. Afterwards, the mixture was diluted with EtOAc and washed with water several times. The organic layer was dried with $\mathrm{Na}_{2} \mathrm{SO}_{4}$, filtered, and evaporated to dryness. The crude product was purified by column chromatography.

General procedure for the glycosylation of BODIPYs $(43,44,47,48$, $51-54)$

The corresponding BODIPY $\mathbf{1 a}, \mathbf{8 a}, \mathbf{2 5}$, or $\mathbf{2 6}$ (1 equiv.) was dissolved in DMF and the corresponding 1 '-thio-carbohydrate sodium salt (1.2 equiv.) was added. The mixture was stirred for the indicated time. Afterwards, $5 \mathrm{~mL}$ water was added and stirred for additional $5 \mathrm{~min}$ at rt. Due to the high polarity of the product, the mixture was directly evaporated to dryness with a rotary evaporator. The crude product was purified by column chromatography and recrystallized.

\section{General procedure for the dibromination of tetramethyl-BODIPYs (34 - 42, 45, 46, 49, and 50)}

The corresponding 1,3,5,7-tetramethyl substituted BODIPY 25 - 33, $\mathbf{4 3}, \mathbf{4 4}, \mathbf{4 7}$, or 48 (1 equiv.) was dissolved in $2 \mathrm{~mL}$ of HFIP, NBS (2.5 equiv.) was added and the mixture was stirred for $1 \mathrm{~min}$ at $\mathrm{rt}$. Afterwards, the reaction mixture was diluted with EtOAc and washed with water several times. The organic layer was dried with $\mathrm{Na}_{2} \mathrm{SO}_{4}$, filtered, and evaporated to dryness. The crude product was purified by column chromatography.

\section{Antibacterial testing}

The bacterium S. aureus is a typical Gram-positive member of the microflora of wounds. Cultured bacterial cells of $S$. aureus ATCC 25923 (WDCM 00034) were suspended in sterile PBS or sterile PBS supplemented with $10 \%$ sterile horse blood serum. The bacterial suspensions were placed into sterile black well plates with clear bottoms. Concentrations of the BODIPYs used in the study were as follows: $100 \mu \mathrm{M}, 10 \mu \mathrm{M}$ and $1 \mu \mathrm{M}$. After an incubation time period of 30 minutes, the samples are exposed to white light (KL 2500 LCD; Schott), with a power density and irradiation time resulting in an energy fluency of about $100 \mathrm{~J} / \mathrm{cm}^{2}$. After irradiation, the samples are removed and suspended again in the culture media. The numbers of colony-forming units (CFU/ml) are enumerated after an adequate incubation time period. Control plates contained no BODIPY and are not exposed to white light. The control samples for dark toxicity are only exposed to BODIPY without any illumination. In an additional control experiment the bacterial suspension was subjected to white light illumination only (see Supporting Information; Figure S15). In the same way as described above the experiments with the Gramnegative bacterium $P$. aeruginosa ATCC 27853 (WDCM 00025) were performed.

\section{X-ray crystallography}

Crystals were grown following the protocol developed by Hope by dissolving the compound in $\mathrm{CH}_{2} \mathrm{Cl}_{2}$ and layering with $\mathrm{MeOH}$ for liquidliquid diffusion. ${ }^{49}$ Single crystal $\mathrm{X}$-ray diffraction data for all compounds were collected on a Bruker APEX 2 DUO CCD diffractometer by using graphite-monochromated $\operatorname{MoK}_{\alpha}(\lambda=0.71073$ $\AA$ ) radiation. Crystals were mounted on a MiTeGen MicroMount and collected at 100 (2) K by using an Oxford Cryosystems Cobra lowtemperature device. Data were collected by using omega and phi scans and were corrected for Lorentz and polarization effects by using the APEX software suite. ${ }^{50}$ The structure was solved with the $\mathrm{XT}$ structure solution program, using the intrinsic phasing solution method and refined against $|F 2|$ with $X L$ using least-squares minimization, using the Olex2 and ShelXle graphical interfaces. ${ }^{51}$ Hydrogen atoms were generally placed in geometrically calculated positions and refined using a riding model unless otherwise stated. All images were rendered using Olex2. ${ }^{51 a}$ Details of data refinements can found in tables S1 in the Supporting Information. Refinement 
details for KJF202: The hydrogen attached to N2 (H2) was allowed to freely refine. The distance of this bond was fixed using the DFIX restraint at 0.88 (0.01) Å. Refinement details for CJK009: N- and Obound $\mathrm{H}$ atoms were allowed to freely refine, with riding isotropic thermal parameters.

CCDC 1975111 (for CJK009) and 1975112 (for KJF202) contains the supplementary crystallographic data for this paper. These data can be obtained free of charge from The Cambridge Crystallographic Data Centre.

\section{Acknowledgments}

This work has received funding from the European Union's Horizon 2020 research and innovation programme under the Marie Skłodowska-Curie Grant Agreement No. 764837 and was supported by a grant from Science Foundation Ireland (IvP 13/IA/1894). The biolitec research $\mathrm{GmbH}$ gratefully acknowledges financial support by the German Bundesministerium für Bildung und Forschung (BMBF) (FKZ 03ZZ0914I and FKZ 03ZZ0927B).

\section{Conflicts of interest}

There are no conflicts to declare.

\section{References}

1 Antimicrobial resistance: global report on surveillance 2014, https://www.who.int/drugresistance/documents/surveillanc ereport/en, (accessed September 2019).

2 (a) N. Kashef, Y-Y. Huang and M. R. Hamblin, Nanophotonics, 2017, 6, 853-879; (b) T. Maisch, Mini-Rev. Med. Chem., 2009, 9, 974-983; (c) M. Wainwright, J. Antimicrob. Chemother., 1998, 42, 13-28; (d) M. R. Hamblin, Curr. Opin. Microbiol., 2016, 33, 67-73.

3 T. Maisch, J. Photochem. Photobiol. B, 2015, 150, 2-10.

4 (a) L. Costa, M. A. F. Faustino, M. G. P. M. S. Neves, Â. Cunha and A. Almeida, Viruses, 2012, 4, 1034-1074; (b) L. Sobotta, P. Skupin-Mrugalska, J. Mielcarek, T. Goslinkski and J. Balzarini, Mini-Rev. Med. Chem., 2015, 15, 503-521; (c) A. Wiehe, J. M. O'Brien and M. O. Senge, Photochem. Photobiol. Sci., 2019, 18, 2565-2612.

5 R. F. Donnelly P. A. McCarron and M. M. Tunney, Microbiol. Res., 2008, 163, 1-12.

6 M. S. Baptista and M. Wainwright, Braz. J. Med. Biol. Res., 2011, 44, 1-10.

7 J. J. T. M. Swartjes, P. K. Sharma, T. G. van Kooten, H. C. van der Mei, M. Mahmmoudi, H. J. Busscher and E. T. J. Rochford, Curr. Med. Chem., 2015, 22, 2116-2129.

8 K. Page, M. Wilson and I. P. Parkin, J. Mater. Chem., 2009, 19, 3819-3831.

9 H. Yazini, M. B. O'Neil, T. Kacar, B. R. Wilson, E. E. Oren, M. Sarikaya and C. Tamerler, ACS Appl. Mater. Interfaces, 2016, 8, 5070-5081.

10 B. Meyer, Int. Biodeterior. Biodegrad., 2003, 51, 249-253.

11 (a) G. Kubheka, I. Uddin, E. Amuhaya, J. Mack and T. Nyokong, J. Porphyrins Phthalocyanines, 2016, 20, 1016-1024; (b) B. L. Carpenter, X. Situ, F. Scholle, J. Bartelmess, W. W. Weare and R. A. Ghiladi, Molecules, 2015, 20, 10604-10621.

12 W. J. Peveler, S. Noimark, A. Al-Azawi, G. B. Hwang, C. R. Crick, E. Allan, J. B. Edel, A. P. Ivanov, A. J. MacRobert and I. P. Parkin, ACS Appl. Mater. Interfaces, 2018, 10, 98-104.
13 (a) N. Boens, V. Leen and W. Dehaen, Chem. Soc. Rev., 2012, 41, 1130-1172; (b) T. Kowada, H. Maeda and K. Kikuchi, Chem. Soc. Rev., 2015, 44, 4953-4972; (c) P. Kaur and K. Singh, J. Mater. Chem. C, 2019, 7, 11361-1405; (d) J. L. Donnelly, D. Offenbartl-Stiegert, J. M. Marín-Beloqui, L. Rizzello, G. Battaglia, T. M. Clarke, S. Howorka and J. D. Wilden, Chem. Eur. J., 2020, 26, 863-872; (e) H. Qi, J. J. Teesdale, R. C. Pupillo, J. Rosenthal and A. J. Bard, J. Am. Chem. Soc., 2013, 135, 13558-13566.

14 A. Kamkaew, S. H. Lim, H. B. Lee, L. V. Kiew, L. Y. Chung and K. Burgess, Chem. Soc. Rev., 2013, 42, 77-88.

15 (a) E. Caruso, S. Banfi, P. Barbieri, B. Leva and V. T. Orlandi, J. Photochem. Photobiol. B, 2012, 114, 44-51; (b) J. H. Gibbs, Z. Zhou, D. Kessel, F. R. Fronczek, S. Pakhomova and M. G. H. Vicente, J. Photochem. Photobiol. B, 2015, 145, 35-47; (c) E. Fron, E. Coutiño-Gonzalez, L. Pandey, M. Sliwa, M. Van der Auweraer, F. C. De Schryver, J. Thomas, Z. Dong, V. Leen, M. Smet, W. Dehaen and T. Vosch, New J. Chem., 2009, 33, 14901496; (d) N. Epelde-Elezcano, V. Martínez-Martínez, E. PeñaCabrera, C. F. A. Gómez-Durán, I. L. Arbeloa and S. Lacombe, RSC Adv., 2016, 6, 41991-41998; (e) N. Boens, B. Verbelen, M. J. Ortiz, L. Jiao and W. Dehaen, Coord. Chem. Rev., 2019, 399, 213024; (f) N. Boens, B. Verbelen and W. Dehaen, Eur. J. Org. Chem., 2015, 6577-6595; (g) A. M. Potocny, J. J. Teesdale, A. Marangoz, G. P. A. Yap and J. Rosenthal, Inorg. Chem., 2019, 58, 5042-5050.

16 G. Fan, L. Yang and Z. Chen, Chem. Sci. Eng., 2014, 8, 405-417.

17 Y. Liu, R. Qin, S. A. J. Zaat, E. Breukink and M. Heger, J. Clin. Translat. Research, 2015, 1, 140-167.

18 (a) M. J. Ortiz, A. R. Agarrabeitia, G. Duran-Sampedro, J. B. Prieto, T. A. Lopez, W. A. Massad, H. A. Montejano, N. A. García and I. P. Arbeloa, Tetrahedron, 2012, 68, 1153-1162; (b) C. F. A. Gómez-Durán, I. Esnal, I. Valois-Escamilla, A. UríasBenavides, J. Bañuelos, I. P. Arbeloa, I. García-Moreno and E. Peña-Cabrera, Chem. Eur. J., 2016, 22, 1048-1061; (c) J. Zou, Z. Yin, K. Ding, Q. Tang, J. Li, W. Si, J. Shao, Q. Zhang, W. Huang and X. Dong, ACS Appl Mater Interfaces, 2017, 9, 3247532481.

19 (a) S. G. Awuah and Y. You, RSC Adv., 2012, 2, 11169-11183; (b) X.-F. Zhang and X. Yang, J. Phys. Chem. B, 2013, 117, 55335539.

20 V. Leen, D. Miscoria, S. Yin, A. Filarowski, J. M. Ngongo, M. Van der Auweraer, N. Boens and W. Dehaen, J. Org. Chem., 2011, 76, 8168-8176.

21 (a) M. A. Filatov, S. Karuthedath, P. M. Polestshuk, H. Savoi, K. J. Flanagan, C. Sy, E. Sitte, M. Telichko, F. Laqaui, R. W. Boyle and M. O. Senge, J. Am. Chem. Soc., 2017, 139, 6282-6285; (b) M. A. Filatov, S. Karuthedath, P. M. Polestshuk, S. Callaghan, K. J. Flanagan, T. Wiesner, F. Laquai and M. O. Senge, ChemPhotoChem, 2018, 2, 606-615; (c) W. Hu, X.-F. Zhang, X. Lu, S. Lan, D. Tian, T. Li, L. Wang, S. Zhao, M. Feng and J. Zhang, Dyes Pigm., 2018, 149, 306-314.

22 (a) G. Jori, C. Fabris, M. Soncin, S. Ferro, O. Coppellotti, D. Dei, L. Fantetti, G. Chiti and G. Roncucci, Lasers Surg. Med., 2006, 38, 468-481; (b) T. Dai, Y.-Y. Huang, S. K. Sharma, J. T. Hashmi, D. B. Kurup and M. R. Hamblin, Recent Patents Anti-Canc. Drug Discov., 2010, 5, 124-151.

23 L. Jiao, W. Pang, J. Zhou, Y. Wei, X. Mu, G. Bai and E. Hao, J. Org. Chem., 2011, 76, 9988-9996.

24 X. Zhou, C. Yu, Z. Feng, Y. Yu, J. Wang, E. Hao, Y. Wei, X. Mu and L. Jiao, Org. Lett., 2015, 17, 4632-4635.

25 L. Wang, J.-W. Wang, A.-j. Cui, X.-X. Cai, Y. Wan, Q. Chen, M.Y. He and W. Zhang, RSC Adv., 2013, 3, 9219-9222.

26 (a) H. R. A. Golf, H.-U. Reissig and A. Wiehe, Org. Lett., 2015, 17, 982-985; (b) C. S. Gutsche, M. Ortwerth, S. Gräfe, K. J. Flanagan, M. O. Senge, H.-U. Reissig, N. Kulak and A. Wiehe, Chem. Eur. J., 2016, 22, 13953-13964; (c) Y. Tian, B. R. Shumway and D. R. Meldrum, Chem. Mater., 2010, 22, 2069- 
2078; (d) S. J. Shaw, K. J. Elgie, C. Edwards and R. W. Boyle, Tetrahedron Lett., 1999, 40, 1595-1596.

27 (a) B. F. Hohlfeld, K. J. Flanagan, N. Kulak, M. O. Senge, M. Christmann and A. Wiehe, Eur. J. Org. Chem., 2019, 40204033; (b) Y. Volkova, B. Brizet, P. D. Harvey, F. Denat and C. Goze, Eur. J. Org. Chem., 2014, 2268-2274.

28 (a) R. Klingenburg, C. B. W. Stark and A. Wiehe, Org. Lett., 2019, 21, 5417-5420; (b) S. Singh, A. Aggarwal, S. Thompson, J. P. C. Tomé, X. Zhu, D. Samaroo, M. Vinodu, R. Gao and C. M. Drain et al., Bioconjugate Chem., 2010, 21, 2136-2146.

29 (a) N. T. Anderson, P. H. Dinolfo and X. Wang, J. Mater. Chem. C, 2018, 6, 2452-2459; (b) M. C Bennion, M. A. Burch, D. G. Dennis, M. E. Lech, K. Neuhaus, N. L. Fendler. M. R. Parris, J. E. Cuadra, C. F. Dixon, G. T. Mukosera, D. N. Blauch. L. Hartmann, N. L. Snyder and J. V. Ruppel, Eur. J. Org. Chem., 2019, 64966503.

30 D. Prasannan, D. Raghav, S. Sujatha, H. Hareendrakrishnakumar, K. Rathinasamy and C. Arunkumar, RSC Adv., 2016, 6, 80808-80824.

31 (a) M. A. H. Alamiry, A. C. Benniston, J. Hagon, T. P. L. Winstanley, H. Lemmityinen and N. V. Tkachenko, RSC Adv. 2012, 2, 4944-4950; (b) B. P. Ngoy, Z. Hlatshwayo, N. Nwaji, G. Fomo, J. Maxk T. Nyokong, J. Porphyrins Phthalocyanines, 2018, 22, 413-422.

32 G. Vives, C. Giansante, R. Bofinger, G. Raffy, A. Del Guerzo, B. Kaufmann, P. Batat, G. Jonusauskas and N. D. McClenaghan, Chem. Commun., 2011, 47, 10425-10427.

33 I. S. Turan, F. P. Cakmak, D. C. Yildirim, R. Cetin-Atalay and E. U. Akkaya et al., Chem. Eur. J., 2014, 20, 16088-16092.

34 (a) K. Jain, P. Kesharwani, U. Gupta and N. K. Jain, Biomaterials, 2012, 33, 4166-4186; (b) H. Zhang, Y. Ma and X.-L. Sun, Med. Res. Rev., 2010, 30, 270-289.

35 (a) E. Cho and S. Jung, Molecules, 2015, 20, 19620-19646; (b) S. V. Moradi, W. M. Hussein, P. Varamini, P. Simerska and I. Toth, Chem. Sci., 2016, 7, 2492-2500.

36 (a) N. Shivran, M. Tyagi, S. Mula, P. Gupta, B. Saha, B. S. Patro and S. Chattopadhyay, Eur. J. Med. Chem., 2016, 122, 352365; (b) T. V. Ovchinnikova, A. V. Pshezhetsky, A. B. Tuzikov and N. V. Bovin, Mendeleev Commun., 2015, 25, 422-423; (c) T. Papalia, A. Barattucci, S. Campagna, F. Puntoriero, T. Salerno and P. Bonaccorsi, Org. Biomol. Chem., 2017, 15 8211-8217.

37 (a) F. Faschinger, S. Aichhorn, M. Himmelsbach and W. Schoefberger, Synthesis, 2014, 46, 3085-3096; (b) D. Samaroo, M. Vinodu, X. Chen and C. M. Drain, J. Comb. Chem. 2007, 9, 998-1011.

38 (a) C. S. Gutsche, S. Gräfe, B. Gitter, K. J. Flanagan, M. O. Senge, N. Kulak and A. Wiehe, Dalton. Trans., 2018, 47, 12373-12384; (b) X. Chen, D. A. Foster and C. M. Drain, Biochemistry, 2004, 43, 10918-10929.

39 P. E. Kesavan, V. Pandey, M. K. Raza, S. Mori and I. Gupta, Bioorg. Chem., 2019, 91, 103139.

40 H. F. Chambers and. F. R. DeLeo, Nat. Rev. Microbiol., 2009, 7, 629-641.

41 T. Dai, Y.-Y. Huang and M. R. Hamblin, Photodiagn. Photodyn. Ther., 2009, 6, 170-188.

42 (a) L.-C. Lu, Y.-W. Chen and C.-C. Chou, Int. J. Food Microbiol., 2005, 102, 213-220; (b) T. E. Shehata and A. G. Marr, J. Bacteriol., 1970, 103, 789-792.

43 M. De Vetta, L. González and I. Corral, ChemPhotoChem. 2019, 3, 727-738.

44 Z. Pang, R. Raudonis, B. R. Glick, T.-J. Lin and Z. Cheng, Biotechnol. Adv., 2019, 37, 177-192.

45 P. Pachori, R. Gothalwal and P. Gandhi, Genes Diseases, 2019 6, 109-119.

46 F. Lucca, M. Guarnieri, M. Ros, G. Muffato, R. Rigoli and L. Da Dalt, Clin. Respir. J., 2018, 12, 2189-2196.
47 (a) B. M. Amos-Tautua, S. P. Songca and O. S. Oluwafemi, Molecules, 2019, 24, 2456; (b) X. Hu, Y.-Y. Huang, Y. Wang, X. Wang and M. R. Hamblin, Front. Microbiol., 2018, 9, 01299; (c) S. M. Yang, D. W. Lee, H. J. Park, M. H. Kwak, J. M. Park and M.-G. Choi, Photochem. Photobiol., 2019, 95, 833-838; (d) Y. Zhao, Z. Lu, X. Dai, X. Wei, Y. Yu, X. Chen, X. Zhang and C. Li, Bioconjugate Chem., 2018, 29, 3222-3230; (e) X. Dai, X. Chen, Y. Zhao, Y. Yu, X. Wei, X. Zhang and C. Li, Biomacromolecules, 2018, 19, 141-149; (f) M. C. E. Hashimoto, R. A. Prates, I. T. Kato, S. C. Núñez, L. C. Courrol and M. S. Ribeiro, Photochem. Photobiol., 2012, 88, 590-595.

48 (a) R. G. Sturmey, C. P. Wild and L. J. Hardie, Mutagenesis, 2009, 24, 253-258; (b) E. Gocke, S. Albertini, A. A. Chételat, S. Kirchner and W. Muster, Toxicol. Lett., 1998, 102-103, 375381.

49 H. Hope, Prog. Inorg. Chem., 2007, 1-19.

50 (a) Saint, Version 8.37a; Bruker AXS, Inc.: Madison, WI, 2013; (b) SADABS, version 2016/2; Bruker AXS, Inc.: Madison, WI, 2014; (c) APEX3, Version 2016.9-0; Bruker AXS, Inc.: Madison, WI, 2016.

51 (a) O. V. Dolomanov, L. J. Bourhis, R. J. Gildea, J. A. K. Howard and H. Puschmann, J. Appl. Cryst., 2009, 42, 339-341; (b) G. Sheldrick, Acta Cryst., 2015, A71, 3-8; (c) C. B. Hübschle, G. M. Sheldrick and B. Dittrich, J. Appl. Cryst., 2011, 44, 1281-1284. 